1 Hacettepe Journal of Mathematics and Statistics

$\bigcap$ Volume 45 (2) (2016), 311-335

\title{
A generalized operational method for solving integro-partial differential equations based on Jacobi polynomials
}

\author{
Abdollah Borhanifar ${ }^{* \dagger}$ and Khadijeh Sadri ${ }^{\ddagger}$
}

\begin{abstract}
In this paper, a numerical method is developed for solving linear and nonlinear integro-partial differential equations in terms of the two variables Jacobi polynomials. First, some properties of these polynomials and several theorems are presented then a generalized approach implementing a collocation method in combination with two dimensional operational matrices of Jacobi polynomials is introduced to approximate the solution of some integro-partial differential equations with initial or boundary conditions. Also, it is shown that the resulted approximate solution is the best approximation for the considered problem. The main advantage is to derive the Jacobi operational matrices of integration and product to achieve the best approximation of the two dimensional integro-differential equations. Numerical results are given to confirm the reliability of the proposed method for solving these equations.
\end{abstract}

Keywords: Best approximation, Collocation method, Integro-partial differential equations, Operational matrix, Shifted Jacobi polynomials.

2000 AMS Classification: 47B36, 14R15, 39B05, 65D15, 15B99, 65R20, 45K005, $47 \mathrm{G} 10,34 \mathrm{~K} 28$.

Received: 18.11.2014 Accepted: 17.02.2015 Doi : 10.15672/HJMS.20164513116

*Department of Mathematics, Faculty of Mathematical Sciences, University of Mohaghegh Ardabili, Ardabil,Iran,

Email: borhani@uma.ac.ir

${ }^{\dagger}$ Corresponding Author

${ }^{\ddagger}$ Department of Mathematics, Faculty of Mathematical Sciences, University of Mohaghegh Ardabili, Ardabil,Iran,

Email: kh.sadri@uma.ac.ir 


\section{Introduction}

Finding the analytical solutions of functional equations has been devoted attention of mathematicians's interest in recent years. Several methods are proposed to achieve this purpose, such as $[7,8,9,10,11,13]$. Many problems in theoretical physics and other sciences lead to integro-partial differential equations. In order to solve these equations, several numerical methods have been proposed such as [21, 22, 25, 26, 28, 29]. The solutions of this kind of equations are often quite complicated. For this reason in many cases, it is required to obtain the approximate solutions. For example the Jacobi collocation method has been applied to solve various differential equations, $[3,14,15,17,18$, 19, 20, 23]. Also, Bhrawy and et al have presented a new Legendre spectral collocation method for fractional Burgers equations, [6]. In [16], authors have used Jacobi-GaussLobatto collocation method for the numerical solution of $1+1$ nonlinear Schrödinger equation. Also, Bhrawy in [1] has presented an pseudospectral approximation based on Jacobi polynomials for generalized Zakharov system. Two spectral tau algorithms based on Jacobi polynomials have been applied to solve multi-term time-space fractional partial differential equations and time fractional diffusion-Wave equations, [4, 5]. Bhrawy and et al have been presented two different collocation scheme for both temporal and spatial discriminations of mobile-immobile advection-dispersion model (TVFO-MIAD model), [2]. Borhanifar and Sadri have utilized a Jacobi operational collocation method for systems of two dimensional integral equations, [12].

In this paper, the Jacobi polynomials are used as a basis function for solving linear and nonlinear integro-partial differential equations, the numerical solution, $u(x, y)$, is approximated in terms of two variables Jacobi polynomials as $x, y \in[0,1]$. In order to realize this aim, the shifted Jacobi polynomials together the collocation technique are used. The Jacobi operational matrices of the integration and product are constructed on the interval $[0,1]$. The main aim is to improve Jacobi operational matrices to the spectral solution of partial integro-differential equations. For solving the resulted algebraic system, the $(N+1)$ roots of one variable Jacobi polynomials $P_{N+1}^{(\alpha, \beta)}(x)$ and $P_{N+1}^{(\alpha, \beta)}(y)$ are considered in the $x, y$-directions. The domain of two dimensional is represented by a tensor product points $\left\{x_{i}\right\}_{i=0}^{N}$ and $\left\{y_{j}\right\}_{j=0}^{N}$ which are roots of $P_{N+1}^{(\alpha, \beta)}(x)$ and $P_{N+1}^{(\alpha, \beta)}(y)$. Each the equations of the algebraic system is collocated in the resulted tensor points $\left\{\left(x_{i}, y_{j}\right)\right\}_{i, j=0}^{N}$ and is given linear or nonlinear systems of algebraic equations which can be solved using the well-known Newtons iterative method. Thus, the Jacobi coefficients are obtained and the approximate solution is determined.

The remainder of this paper is organized as follows: The Jacobi polynomials, some of their properties and one dimensional operational matrix of integration are introduced in Section 2. Afterwards, some properties of two variables Jacobi polynomials are stated and the operational matrices of integration and product will be extended to two dimensional case in Section 3. In Section 4, the existence and uniqueness of the best approximation are studied and an error estimator is introduced. Section 5 is devoted to applying two dimensional Jacobi operational matrices for solving the partial integro-differential equations. For this purpose, four examples are presented. A conclusion is presented in Section 6.

\section{Jacobi polynomials and their operational matrix of integration}

The well-known Jacobi polynomials are defined on the interval $z \in[-1,1]$, constitute an orthogonal system with respect to the weight function $w^{(\alpha, \beta)}(z)=(1-z)^{\alpha}(1+z)^{\beta}$, 
and can be determined with the following recurrence formula:

$$
\begin{gathered}
P_{i+1}^{(\alpha, \beta)}(z)=A(\alpha, \beta, i) P_{i}^{(\alpha, \beta)}(z)+z B(\alpha, \beta, i) P_{i}^{(\alpha, \beta)}(z)-D(\alpha, \beta, i) P_{i-1}^{(\alpha, \beta)}(z), \\
i=1,2, \ldots,
\end{gathered}
$$

where

$$
\begin{aligned}
& A(\alpha, \beta, i)=\frac{(2 i+\alpha+\beta+1)\left(\alpha^{2}-\beta^{2}\right)}{2(i+1)(i+\alpha+\beta+1)(2 i+\alpha+\beta)}, \\
& B(\alpha, \beta, i)=\frac{(2 i+\alpha+\beta+2)(2 i+\alpha+\beta+1)}{2(i+1)(i+\alpha+\beta+1)}, \\
& D(\alpha, \beta, i)=\frac{(i+\alpha)(i+\beta)(2 i+\alpha+\beta+2)}{(i+1)(i+\alpha+\beta+1)(2 i+\alpha+\beta)},
\end{aligned}
$$

and

$$
P_{0}^{(\alpha, \beta)}(z)=1, \quad P_{1}^{(\alpha, \beta)}(z)=\frac{\alpha+\beta+2}{2} z+\frac{\alpha-\beta}{2} .
$$

The orthogonality condition of Jacobi polynomials is

$$
\int_{-1}^{1} P_{j}^{(\alpha, \beta)}(z) P_{k}^{(\alpha, \beta)}(z) w^{(\alpha, \beta)}(z) d z=h_{k} \delta_{j k},
$$

where

$$
h_{k}=\frac{2^{\alpha+\beta+1} \Gamma(k+\alpha+1) \Gamma(k+\beta+1)}{(2 k+\alpha+\beta+1) k ! \Gamma(k+\alpha+\beta+1)} .
$$

The analytic form of Jacobi polynomials is given by, [27],

$$
\begin{aligned}
P_{i}^{(\alpha, \beta)}(z) & =\sum_{k=0}^{i} \frac{(-1)^{(i-k)} \Gamma(i+\beta+1) \Gamma(i+k+\alpha+\beta+1)}{\Gamma(k+\beta+1) \Gamma(i+\alpha+\beta+1)(i-k) ! k !}\left(\frac{1+z}{2}\right)^{k}, \\
i & =0,1, \ldots .
\end{aligned}
$$

For practical use of Jacobi polynomials on the interval $x \in[0,1]$, it is necessary to shift the defining domain by means of the following change variable:

$$
z=2 x-1, \quad x \in[0,1]
$$

The shifted Jacobi polynomials are generated from the three-term recurrence relation

$$
\begin{gathered}
P_{i+1}^{(\alpha, \beta)}(x)=A(\alpha, \beta, i) P_{i}^{(\alpha, \beta)}(x)+(2 x-1) B(\alpha, \beta, i) P_{i}^{(\alpha, \beta)}(x) \\
-D(\alpha, \beta, i) P_{i-1}^{(\alpha, \beta)}(x), \quad i=1,2, \ldots, \\
P_{0}^{(\alpha, \beta)}(x)=1, \quad P_{1}^{(\alpha, \beta)}(x)=\frac{(\alpha+\beta+2)(2 x-1)}{2}+\frac{\alpha-\beta}{2} .
\end{gathered}
$$

The orthogonality condition and weight function are respectively,

$$
\int_{0}^{1} P_{i}^{(\alpha, \beta)}(x) P_{j}^{(\alpha, \beta)}(x) w^{(\alpha, \beta)}(x) d x=\theta_{i} \delta_{i j},
$$

where

$$
\theta_{i}=\frac{\Gamma(i+\alpha+1) \Gamma(i+\beta+1)}{(2 i+\alpha+\beta+1) i ! \Gamma(i+\alpha+\beta+1)}
$$

and

$$
w^{(\alpha, \beta)}(x)=(1-x)^{\alpha} x^{\beta}, \quad x \in[0,1] .
$$


Also, the analytic form of shifted Jacobi polynomials will be as follows, [27],

$$
\begin{aligned}
P_{i}^{(\alpha, \beta)}(x) & =\sum_{k=0}^{i} \frac{(-1)^{(i-k)} \Gamma(i+\beta+1) \Gamma(i+k+\alpha+\beta+1) x^{k}}{\Gamma(k+\beta+1) \Gamma(i+\alpha+\beta+1)(i-k) ! k !}, \\
i & =0,1, \ldots .
\end{aligned}
$$

A continuous function $u(x)$, square integrable in $[0,1]$, can be expressed in terms of shifted Jacobi polynomials as

$$
u(x)=\sum_{j=0}^{\infty} C_{j} P_{j}^{(\alpha, \beta)}(x),
$$

where the coefficients $C_{j}$ are given by

$$
C_{j}=\frac{1}{\theta_{j}} \int_{0}^{1} u(x) P_{j}^{(\alpha, \beta)}(x) w^{(\alpha, \beta)}(x) d x, \quad j=0,1, \ldots .
$$

In practice, only the first $(N+1)$-terms shifted Jacobi polynomials are considered. Therefore, one has

$$
u_{N}(x)=\sum_{j=0}^{N} C_{j} P_{j}^{(\alpha, \beta)}(x)=\Phi^{T}(x) C=C^{T} \Phi(x),
$$

where the vectors $C$ and $\Phi(x)$ are given by

$$
C=\left[C_{0}, C_{1}, \ldots, C_{N}\right]^{T}, \quad \Phi(x)=\left[P_{0}^{(\alpha, \beta)}(x), P_{1}^{(\alpha, \beta)}(x), \ldots, P_{N}^{(\alpha, \beta)}(x)\right]^{T} .
$$

Some other properties of the shifted Jacobi polynomials are presented as follows.

$$
\begin{aligned}
& \text { (1) } P_{i}^{(\alpha, \beta)}(0)=(-1)^{i}\left(\begin{array}{c}
i+\alpha \\
i
\end{array}\right) \\
& \text { (2) } \frac{d^{i}}{d x^{i}} P_{n}^{(\alpha, \beta)}(x)=\frac{\Gamma(n+\alpha+\beta+i+1)}{\Gamma(n+\alpha+\beta+1)} P_{n-i}^{(\alpha+i, \beta+i)}(x) .
\end{aligned}
$$

2.1. Lemma. The shifted Jacobi polynomial $P_{i}^{(\alpha, \beta)}(x)$ can be obtained in the form of:

$$
P_{i}^{(\alpha, \beta)}(x)=\sum_{k=0}^{i} \gamma_{k}^{(i)} x^{k}
$$

where $\gamma_{k}^{(i)}$ are

$$
\gamma_{k}^{(i)}=(-1)^{i-k}\left(\begin{array}{c}
i+k+\alpha+\beta \\
k
\end{array}\right)\left(\begin{array}{c}
i+\alpha \\
i-k
\end{array}\right) .
$$

Proof. $\gamma_{k}^{(i)}$ can be obtained as,

$$
\gamma_{k}^{(i)}=\left.\frac{1}{k !} \frac{d^{k}}{d x^{k}} P_{i}^{(\alpha, \beta)}(x)\right|_{x=0} .
$$

Now, using properties (1) and (2), the lemma can be proved.

2.2. Lemma. If $p>\beta-1$, then

$$
\begin{aligned}
& \int_{0}^{1} x^{p-\beta} P_{n}^{(\alpha, \beta)}(x) w^{(\alpha, \beta)}(x) d x= \\
& \quad \sum_{l=0}^{n} \frac{(-1)^{n-l} \Gamma(n+\beta+1) \Gamma(n+l+\alpha+\beta+1) \Gamma(p+l+1) \Gamma(\alpha+1)}{\Gamma(l+\beta+1) \Gamma(n+\alpha+\beta+1) \Gamma(p+l+\alpha+2)(n-l) ! l !} .
\end{aligned}
$$


Proof. For $p-\beta<n$ one has

$$
\int_{0}^{1} x^{p-\beta} P_{n}^{(\alpha, \beta)}(x) w^{(\alpha, \beta)}(x) d x=0 .
$$

Hence, we suppose $p-\beta \geq n$. From analytic form of shifted Jacobi polynomials, (2.3), one has

$$
\begin{aligned}
\int_{0}^{1} x^{p-\beta} & P_{n}^{(\alpha, \beta)}(x) w^{(\alpha, \beta)}(x) d x= \\
& \sum_{l=0}^{n} \frac{(-1)^{n-l} \Gamma(n+\beta+1) \Gamma(n+l+\alpha+\beta+1)}{\Gamma(l+\beta+1) \Gamma(n+\alpha+\beta+1)(n-l) ! l !} B(p+l+1, \alpha+1) \\
& =\sum_{l=0}^{n} \frac{(-1)^{n-l} \Gamma(n+\beta+1) \Gamma(n+l+\alpha+\beta+1) \Gamma(p+l+1) \Gamma(\alpha+1)}{\Gamma(l+\beta+1) \Gamma(n+\alpha+\beta+1) \Gamma(p+l+\alpha+2)(n-l) ! l !},
\end{aligned}
$$

where $B(s, t)$ is the Beta function and is defined as

$$
B(s, t)=\int_{0}^{1} v^{s-1}(1-v)^{t-1} d v=\frac{\Gamma(s) \Gamma(t)}{\Gamma(s+t)} .
$$

2.3. Lemma. If $P_{j}^{(\alpha, \beta)}(x)$ and $P_{k}^{(\alpha, \beta)}(x)$ are $j$-th and $k$-th shifted Jacobi polynomials, respectively, then the product of $P_{j}^{(\alpha, \beta)}(x)$ and $P_{k}^{(\alpha, \beta)}(x)$ can be written as

$$
Q_{j+k}^{(\alpha, \beta)}(x)=\sum_{r=0}^{j+k} \lambda_{r}^{(j, k)} x^{r}
$$

Proof. Defining the $Q_{j+k}^{(\alpha, \beta)}(x)=P_{j}^{(\alpha, \beta)}(x) P_{k}^{(\alpha, \beta)}(x)$ as a polynomial of degree $j+k$ that can be written as

$$
Q_{j+k}^{(\alpha, \beta)}(x)=\left(\sum_{m=0}^{j} \gamma_{m}^{(j)} x^{m}\right)\left(\sum_{n=0}^{k} \gamma_{n}^{(k)} x^{n}\right)=\sum_{r=0}^{j+k} \lambda_{r}^{(j, k)} x^{r} .
$$

The relation between coefficients $\lambda_{n}^{(j, k)}$ with coefficients $\gamma_{m}^{(j)}$ and $\gamma_{m}^{(k)}$ will be as follows.

$\underline{\underline{\text { If } j \geq k:}}$

$$
\begin{aligned}
& r=0,1, \ldots, j+k, \\
& \text { if } r>j \text { then } \\
& \quad \lambda_{r}^{(j, k)}=\sum_{l=r-j}^{k} \gamma_{r-l}^{(j)} \gamma_{l}^{(k)}, \\
& \text { else } \quad r_{1}=\min \{r, k\}, \\
& \quad \lambda_{r}^{(j, k)}=\sum_{l=0}^{r_{1}} \gamma_{r-l}^{(j)} \gamma_{l}^{(k)}, \\
& \text { end. }
\end{aligned}
$$

$\underline{\underline{\text { If } j<k:}}$

$$
\begin{aligned}
& r=0,1, \ldots, j+k, \\
& \text { if } r \leq j \text { then } \\
& \quad r_{1}=\min \{r, j\}, \\
& \quad \lambda_{r}^{(j, k)}=\sum_{l=0}^{r_{1}} \gamma_{r-l}^{(j)} \gamma_{l}^{(k)}, \\
& \text { else } \\
& \quad r_{2}=\min \{r, k\}, \\
& \quad \lambda_{r}^{(j, k)}=\sum_{l=r-j}^{r_{2}} \gamma_{r-l}^{(j)} \gamma_{l}^{(k)}, \\
& \text { end. }
\end{aligned}
$$


Thus, the coefficients $\lambda_{r}^{(j, k)}$ is determined.

2.4. Lemma. If $P_{i}^{(\alpha, \beta)}(x), P_{j}^{(\alpha, \beta)}(x)$ and $P_{k}^{(\alpha, \beta)}(x)$ are $i-, j-$ and $k$-th shifted Jacobi polynomials, respectively, then

$\int_{0}^{1} P_{i}^{(\alpha, \beta)}(x) P_{j}^{(\alpha, \beta)}(x) P_{k}^{(\alpha, \beta)}(x) w^{(\alpha, \beta)}(x) d x=$

$\sum_{n=0}^{j+k} \sum_{l=0}^{i} \frac{(-1)^{i-l} \lambda_{n}^{(j, k)} \Gamma(i+\beta+1) \Gamma(i+l+\alpha+\beta+1) \Gamma(n+l+\beta+1) \Gamma(\alpha+1)}{\Gamma(l+\beta+1) \Gamma(i+\alpha+\beta+1) \Gamma(n+l+\alpha+\beta+2)(i-l) ! l !}$,

where $\lambda_{n}^{(j, k)}$ has been introduced in Lemma 2.3.

Proof. Assuming that $P_{j}^{(\alpha, \beta)}(x) P_{k}^{(\alpha, \beta)}(x)=Q_{j+k}^{(\alpha, \beta)}(x)$. Using of (2.3), Lemmas 2.2 and 2.3 leads to

$$
\begin{aligned}
& \int_{0}^{1} P_{i}^{(\alpha, \beta)}(x) P_{j}^{(\alpha, \beta)}(x) P_{k}^{(\alpha, \beta)}(x) w^{(\alpha, \beta)}(x) d x= \\
& \int_{0}^{1} P_{i}^{(\alpha, \beta)}(x) Q_{j+k}^{(\alpha, \beta)}(x) w^{(\alpha, \beta)}(x) d x \\
& =\sum_{n=0}^{j+k} \lambda_{n}^{(j, k)} \int_{0}^{1} x^{n} P_{i}^{(\alpha, \beta)}(x) w^{(\alpha, \beta)}(x) d x \\
& =\sum_{n=0}^{j+k} \sum_{l=0}^{i} \frac{(-1)^{i-l} \Gamma(i+\beta+1) \Gamma(i+l+\alpha+\beta+1)}{\Gamma(l+\beta+1) \Gamma(i+\alpha+\beta+1)(i-l) ! l !} B(n+l+\beta+1, \alpha+1),
\end{aligned}
$$

Thus, desirable result is obtained.

In performing arithmetic and other operations on the Jacobi bases, we frequently encounter the integration of the vector $\Phi(x)$ defined in (2.6) which is called the operational matrix of the integration. Hence, the matrix relations must be obtained. In this section, this operational matrix will be derived, then it will be extended to two dimensional case in next section. To this end, some useful lemmas and theorems are stated.

2.5. Theorem. Let $\Phi(x)$ be shifted Jacobi vector in (2.6). Then

$$
\int_{0}^{x} \Phi(t) d t \simeq P \Phi(x)
$$

where $P$ is the $(N+1) \times(N+1)$ operational matrix of the integration and is defined by:

$$
P=\left[\begin{array}{cccc}
\Omega(0,0) & \Omega(0,1) & \ldots & \Omega(0, N) \\
\Omega(1,0) & \Omega(1,1) & \ldots & \Omega(1, N) \\
\vdots & \vdots & \ddots & \vdots \\
\Omega(N, 0) & \Omega(N, 1) & \ldots & \Omega(N, N)
\end{array}\right]
$$

where

$$
\Omega(i, j)=\sum_{l=0}^{i} \omega_{i j l}, \quad i, j=0,1, \ldots, N
$$


and $\omega_{i j l}$ are given by

$$
\begin{aligned}
\omega_{i j l}= & \frac{(-1)^{i-l} \Gamma(i+\beta+1) \Gamma(i+l+\alpha+\beta+1)}{\Gamma(l+\beta+1) \Gamma(i+\alpha+\beta+1)(l+1) !(i-l) !} \\
& \times \sum_{k=0}^{j} \frac{(-1)^{j-k} \Gamma(j+k+\alpha+\beta+1) \Gamma(j+\beta+1) \Gamma(k+l+\beta+2) \Gamma(\alpha+1)}{\theta_{j} \Gamma(k+\beta+1) \Gamma(j+\alpha+\beta+1) \Gamma(k+l+\alpha+\beta+3) k !(j-k) !}, \\
& \quad i, j=0,1, \ldots, N, \quad l=0,1, \ldots, i .
\end{aligned}
$$

Proof. Integrating the analytical form of $P_{i}^{(\alpha, \beta)}(x)$, i.e. $(2.3)$, from 0 to $x$ leads to

$$
\int_{0}^{x} P_{i}^{(\alpha, \beta)}(t) d t=\sum_{l=0}^{i} \frac{(-1)^{i-l} \Gamma(i+\beta+1) \Gamma(i+l+\alpha+\beta+1) x^{l+1}}{\Gamma(l+\beta+1) \Gamma(i+\alpha+\beta+1)(l+1) !(i-l) !} .
$$

Now, one can approximate $x^{l+1}$ in terms of shifted Jacobi polynomials as

$$
x^{l+1}=\sum_{k=0}^{N} a_{l, j} P_{j}^{(\alpha, \beta)}(x),
$$

where

$$
a_{l, j}=\frac{1}{\theta_{j}} \int_{0}^{1} x^{l+1} P_{j}^{(\alpha, \beta)}(x) w^{(\alpha, \beta)}(x) d x .
$$

But according to Lemma 2.2 one has,

$$
\begin{aligned}
& \int_{0}^{1} x^{l+1} P_{j}^{(\alpha, \beta)}(x) w^{(\alpha, \beta)}(x) d x= \\
& \sum_{k=0}^{j} \frac{(-1)^{(j-k)} \Gamma(j+k+\alpha+\beta+1) \Gamma(j+\beta+1) \Gamma(k+l+\beta+2) \Gamma(\alpha+1)}{\Gamma(k+\beta+1) \Gamma(j+\alpha+\beta+1) \Gamma(k+l+\alpha+\beta+3) k !(j-k) !} .
\end{aligned}
$$

Therefore, (2.10) will be as follows:

$$
\begin{aligned}
& \int_{0}^{x} P_{i}^{(\alpha, \beta)}(t) d t=\sum_{j=0}^{N}\left\{\sum_{l=0}^{i} \frac{(-1)^{i-l} \Gamma(i+\beta+1) \Gamma(i+l+\alpha+\beta+1)}{\Gamma(l+\beta+1) \Gamma(i+\alpha+\beta+1)(l+1) !(i-l) !}\right. \\
& \left.\quad \times \sum_{k=0}^{j} \frac{(-1)^{j-k} \Gamma(j+k+\alpha+\beta+1) \Gamma(j+\beta+1) \Gamma(k+l+\beta+2)}{\theta_{j} \Gamma(k+\beta+1) \Gamma(j+\alpha+\beta+1) \Gamma(k+l+\alpha+\beta+3)(j-k) !(k) !}\right\} \\
& \quad \times P_{j}^{(\alpha, \beta)}(x) \\
& =\sum_{j=0}^{N} \Omega(i, j) P_{j}^{(\alpha, \beta)}(x) .
\end{aligned}
$$

where $\Omega(i, j)$ are given in (2.9). Accordingly, rewriting (2.11) as a vector form gives

$$
\int_{0}^{x} P_{i}^{(\alpha, \beta)}(t) d t=[\Omega(i, 0), \Omega(i, 1), \ldots, \Omega(i, N)] \Phi(x), \quad i=0,1, . ., N .
$$

This leads to the desired result.

\section{Two variables Jacobi polynomials and their operational matri- ces}

Now in this section, two variables Jacobi polynomials can be defined by means of one variable Jacobi polynomials as follows: 
3.1. Definition. Let $\left\{P_{n}^{(\alpha, \beta)}(x)\right\}_{n=0}^{\infty}$ be the sequence of one variable shifted Jacobi polynomials on $D=[0,1]$. Two variables Jacobi polynomials, $\left\{P_{m, n}^{(\alpha, \beta)}(x, y)\right\}_{m, n=0}^{\infty}$ are defined on $D^{2}=[0,1] \times[0,1]$ as:

$$
P_{m, n}^{(\alpha, \beta)}(x, y)=P_{m}^{(\alpha, \beta)}(x) P_{n}^{(\alpha, \beta)}(y), \quad(x, y) \in D^{2} .
$$

The family $\left\{P_{m, n}^{(\alpha, \beta)}(x, y)\right\}_{m, n=0}^{\infty}$ is orthogonal with weighted function $w^{(\alpha, \beta)}(x, y)=w^{(\alpha, \beta)}(x) w^{(\alpha, \beta)}(y)$ on $D^{2}$ and forms a basis for $L^{2}\left(D^{2}\right)$.

3.2. Theorem. The basis $\left\{P_{m, n}^{(\alpha, \beta)}(x, y)\right\}_{m, n=0}^{\infty}$ is orthogonal on $D^{2}$.

Proof. One has

$$
\begin{aligned}
\int_{0}^{1} \int_{0}^{1} P_{m, n}^{(\alpha, \beta)}(x, y) & P_{k, l}^{(\alpha, \beta)}(x, y) w^{(\alpha, \beta)}(x, y) d x d y= \\
& \int_{0}^{1} P_{m}^{(\alpha, \beta)}(x) P_{k}^{(\alpha, \beta)}(x) w^{(\alpha, \beta)}(x) d x \\
& \times \int_{0}^{1} P_{n}^{(\alpha, \beta)}(y) P_{l}^{(\alpha, \beta)}(y) w^{(\alpha, \beta)}(y) d y \\
& =\left\{\begin{array}{cc}
\theta_{m} \theta_{n}, & (m, n)=(k, l), \\
0, & (m, n) \neq(k, l) \text { or } m \neq k \text { or } n \neq l .
\end{array}\right.
\end{aligned}
$$

Similar to one variable case, a two variables continuous function $u(x, y)$ defined over $D^{2}$ may be expanded by the two variables Jacobi polynomials as:

$$
u(x, y)=\sum_{i=0}^{\infty} \sum_{j=0}^{\infty} C_{i j} P_{i, j}^{(\alpha, \beta)}(x, y)
$$

where the Jacobi coefficients, $C_{i j}$, are obtained as:

$$
C_{i j}=\frac{1}{\theta_{i} \theta_{j}} \int_{0}^{1} \int_{0}^{1} P_{i, j}^{(\alpha, \beta)}(x, y) u(x, y) w^{(\alpha, \beta)}(x, y) d x d y .
$$

If the infinite series in (3.2) is truncated up to their $(N+1)$-terms in terms of both two variables $x$ and $y$ then it can be written as:

$$
u(x, y) \simeq u_{N}(x, y)=\sum_{i=0}^{N} \sum_{j=0}^{N} C_{i j} P_{i, j}^{(\alpha, \beta)}(x, y)=\Phi^{T}(x, y) C,
$$

where $C$ and $\Phi(x, y)$ are Jacobi coefficients and Jacobi polynomials vectors, respectively:

$$
\begin{gathered}
C=\left[C_{00}, C_{01}, \ldots, C_{0 N}, C_{10}, \ldots, C_{1 N}, \ldots, C_{N 1}, \ldots, C_{N N}\right]^{T}, \\
\Phi(x, y)=\left[P_{0,0}^{(\alpha, \beta)}(x, y), P_{0,1}^{(\alpha, \beta)}(x, y), \ldots, P_{0, N}^{(\alpha, \beta)}(x, y), P_{1,0}^{(\alpha, \beta)}(x, y),\right. \\
\left.\ldots, P_{1, N}^{(\alpha, \beta)}(x, y), \ldots, P_{N, 0}^{(\alpha, \beta)}(x, y), \ldots, P_{N, N}^{(\alpha, \beta)}(x, y)\right]^{T} .
\end{gathered}
$$

A function of four variables, $k(x, y, t, s)$, on $D^{4}$ may be approximated based on Jacobi operational matrix as:

$$
k(x, y, t, s) \simeq \Phi^{T}(x, y) K \Phi(t, s),
$$

where $\Phi(x, y)$ is two variables Jacobi vector defined by (3.4) and $K$ is a $(N+1)^{2} \times(N+1)^{2}$ known matrix. Before proceeding, let us represent the partial series (3.3) as following form:

$$
S_{N}(x, y)=\sum_{i=0}^{M} d_{i} Q_{i}^{(\alpha, \beta)}(x, y)
$$


where

$$
\begin{aligned}
& d_{i}=C_{r s}, \quad Q_{i}(x, y)=P_{r, s}^{(\alpha, \beta)}(x, y), \\
& r=\left\lfloor\frac{i}{N+1}\right\rfloor, \quad s=i-r(N+1), \quad M=(N+1)^{2}-1 .
\end{aligned}
$$

Hence, one has

$$
\begin{aligned}
& \int_{0}^{1} \int_{0}^{1} Q_{k}^{(\alpha, \beta)}(x, y) Q_{l}^{(\alpha, \beta)}(x, y) w^{(\alpha, \beta)}(x, y) d x d y= \\
& \int_{0}^{1} P_{\left\lfloor\frac{k}{N+1}\right\rfloor}^{(\alpha, \beta)}(x) P_{\left\lfloor\frac{l}{N+1}\right\rfloor}^{(\alpha, \beta)}(x) w^{(\alpha, \beta)}(x) d x \\
& \times \int_{0}^{1} P_{k-\left\lfloor\frac{k}{N+1}\right\rfloor(N+1)}^{(\alpha, \beta)}(y) P_{l-\left\lfloor\frac{l}{N+1}\right\rfloor(N+1)}^{(\alpha, \beta)}(y) w^{(\alpha, \beta)}(y) d y \\
& =\theta_{\left\lfloor\frac{k}{N+1}\right\rfloor} \theta_{k-\left\lfloor\frac{k}{N+1}\right\rfloor(N+1)}=\theta_{r} \theta_{s}, \quad r=\left\lfloor\frac{k}{N+1}\right\rfloor, \quad s=k-r(N+1) .
\end{aligned}
$$

3.3. Remark. The relation (3.5) can be rewritten as:

$$
S_{N}(x, y)=\sum_{i=0}^{M} \omega_{i} R_{i}^{(\alpha, \beta)}(x, y),
$$

where

$\omega_{i}=d_{i} \sqrt{\theta_{r} \theta_{s}}, \quad R_{i}^{(\alpha, \beta)}(x, y)=\frac{Q_{i}^{(\alpha, \beta)}(x, y)}{\sqrt{\theta_{r} \theta_{s}}}, \quad r=\left\lfloor\frac{i}{N+1}\right\rfloor, \quad s=i-r(N+1)$.

This shows the sequence $\left\{R_{i}^{(\alpha, \beta)}(x, y)\right\}_{i=0}^{M}$ is orthonormal. That is

$$
\int_{0}^{1} \int_{0}^{1} R_{k}^{(\alpha, \beta)}(x, y) R_{l}^{(\alpha, \beta)}(x, y) w^{(\alpha, \beta)}(x, y) d x d y= \begin{cases}1, & k=l \\ 0, & \text { otherwise }\end{cases}
$$

Now, the two dimensional operational matrices of integration in $x$ and $y$-direction are defined by following theorem:

3.4. Theorem. If $P$ is the operational matrix in one dimensional case then the operational matrices of integration in $x$ and $y$-direction are defined as follows.
a) $\int_{0}^{x} \Phi(t, y) d t \simeq P_{x} \Phi(x, y)=(P \otimes I) \Phi(x, y)$,
b) $\int_{0}^{y} \Phi(x, s) d s \simeq P_{y} \Phi(x, y)=(I \otimes P) \Phi(x, y)$,

where $P_{x}$ and $P_{y}$ are $(N+1)^{2} \times(N+1)^{2}$ operational matrices of integration in the directions $x$ and $y$, respectively, $I$ is $(N+1) \times(N+1)$ identity matrix and $\otimes$ denotes the Kronecker product and is defined for two arbitrary matrices $A$ and $B$ as $A \otimes B=\left(a_{i j} B\right)$.

Proof. a) Suppose $r_{j}$ be $j$ th row of matrix $P$. One has

$$
\int_{0}^{x} P_{j}^{(\alpha, \beta)}(t) d t=r_{j}^{T} \Phi(x) .
$$

Also, noting the definition of the vector $\Phi(x, y)$ one has

$$
\begin{aligned}
\Phi(x, y) & =\left[P_{0}^{(\alpha, \beta)}(x) P_{0}^{(\alpha, \beta)}(y), \ldots, P_{0}^{(\alpha, \beta)}(x) P_{N}^{(\alpha, \beta)}(y),\right. \\
& \left.\ldots, P_{N}^{(\alpha, \beta)}(x) P_{0}^{(\alpha, \beta)}(y), \ldots, P_{N}^{(\alpha, \beta)}(x) P_{N}^{(\alpha, \beta)}(y)\right]^{T} .
\end{aligned}
$$


Integrating of (3.7) from 0 to $x$ yields

$$
\begin{aligned}
& \int_{0}^{x} \Phi(t, y) d t=\left[P_{0}^{(\alpha, \beta)}(y) \int_{0}^{x} P_{0}^{(\alpha, \beta)}(t) d t, \ldots, P_{N}^{(\alpha, \beta)}(y) \int_{0}^{x} P_{0}^{(\alpha, \beta)}(t) d t,\right. \\
& \left.\ldots, P_{N}^{(\alpha, \beta)}(y) \int_{0}^{x} P_{N}^{(\alpha, \beta)}(t) d t\right]^{T} \\
& =\left[r_{0} . \Phi(x) P_{0}^{(\alpha, \beta)}(y), \ldots, r_{0} . \Phi(x) P_{N}^{(\alpha, \beta)}(y), \ldots, r_{N} . \Phi(x) P_{0}^{(\alpha, \beta)}(y),\right. \\
& \left.\ldots, r_{N} . \Phi(x) P_{N}^{(\alpha, \beta)}(y)\right]^{T} \\
& =\left[r_{0}\left[P_{0}^{(\alpha, \beta)}(x) P_{0}^{(\alpha, \beta)}(y), \ldots, P_{N}^{(\alpha, \beta)}(x) P_{0}^{(\alpha, \beta)}(y)\right],\right. \\
& \left.\ldots, r_{N}\left[P_{0}^{(\alpha, \beta)}(x) P_{N}^{(\alpha, \beta)}(y), \ldots, P_{N}^{(\alpha, \beta)}(x) P_{N}^{(\alpha, \beta)}(y)\right]\right]^{T} \\
& =\left[\begin{array}{ccccccccccccc}
P_{00} & 0 & \ldots & 0 & P_{01} & 0 & \ldots & 0 & \ldots & P_{0 N} & 0 & \ldots & 0 \\
0 & P_{00} & \ldots & 0 & 0 & P_{01} & \ldots & 0 & \ldots & 0 & P_{0 N} & \ldots & 0 \\
\vdots & \vdots & \ddots & \vdots & \vdots & \vdots & \ddots & \vdots & \ddots & \vdots & \vdots & \ddots & 0 \\
0 & 0 & \ldots & P_{00} & 0 & 0 & \ldots & P_{01} & \ldots & 0 & 0 & \ldots & P_{0 N} \\
& \vdots & & & & \vdots & & & & \vdots & & & \\
P_{N 0} & 0 & \ldots & 0 & P_{N 1} & 0 & \ldots & 0 & \ldots & P_{N N} & 0 & \ldots & 0 \\
0 & P_{N 0} & \ldots & 0 & 0 & P_{N 1} & \ldots & 0 & \ldots & 0 & P_{N N} & \ldots & 0 \\
\vdots & \vdots & \ddots & \vdots & \vdots & \vdots & \ddots & \vdots & \ddots & \vdots & \vdots & \ddots & \vdots \\
0 & 0 & \ldots & P_{N 0} & 0 & 0 & \ldots & P_{N 1} & \ldots & 0 & 0 & \ldots & P_{N N}
\end{array}\right] \\
& \times\left[\begin{array}{c}
P_{0}^{(\alpha, \beta)}(x) P_{0}^{(\alpha, \beta)}(y) \\
P_{0}^{(\alpha, \beta)}(x) P_{1}^{(\alpha, \beta)}(y) \\
\vdots \\
P_{0}^{(\alpha, \beta)}(x) P_{N}^{(\alpha, \beta)}(y) \\
\vdots \\
P_{N}^{(\alpha, \beta)}(x) P_{0}^{(\alpha, \beta)}(y) \\
P_{N}^{(\alpha, \beta)}(x) P_{1}^{(\alpha, \beta)}(y) \\
\vdots \\
P_{N}^{(\alpha, \beta)}(x) P_{N}^{(\alpha, \beta)}(y)
\end{array}\right] \\
& =\left[\begin{array}{cccc}
P_{00} I & P_{01} I & \ldots & P_{0 N} I \\
P_{10} I & P_{11} I & \ldots & P_{1 N} I \\
\vdots & \vdots & \ddots & \vdots \\
P_{N 0} I & P_{N 1} I & \ldots & P_{N N} I
\end{array}\right]\left[\begin{array}{c}
P_{0}^{(\alpha, \beta)}(x) \Phi(y) \\
P_{1}^{(\alpha, \beta)}(x) \Phi(y) \\
\vdots \\
P_{N}^{(\alpha, \beta)}(x) \Phi(y)
\end{array}\right] \\
& =(P \otimes I) \Phi(x, y) \text {. }
\end{aligned}
$$

Where $P_{i j}$ denotes $(i, j)$-th entry of the matrix $P$. The case $(b)$ is proven similarly.

The following property of the product of two vectors $\Phi(x, y)$ and $\Phi^{T}(x, y)$ will also be used.

(3.8) $\Phi(x, y) \Phi^{T}(x, y) C \simeq \tilde{C} \Phi(x, y)$,

where $C$ and $\tilde{C}$ are a $(N+1)^{2} \times 1$ vector and a $(N+1)^{2} \times(N+1)^{2}$ operational matrix of product, respectively. 
3.5. Theorem. The entries of the matrix $\tilde{C}$, in (3.8), are computed as:

$$
\tilde{C}_{m(N+1)+n, k(N+1)+l}=\frac{1}{\theta_{k} \theta_{l}} \sum_{r=0}^{N} \sum_{s=0}^{N} C_{r s} q_{m r k} q_{n s l}, \quad m, n, k, l=0,1, \ldots, N .
$$

Proof. The left side of equality (3.8) is as follows:

$$
\Phi(x, y) \Phi^{T}(x, y) C=\left[\begin{array}{c}
\sum_{r=0}^{N} \sum_{s=0}^{N} C_{r s} P_{0,0}^{(\alpha, \beta)}(x, y) P_{r, s}^{(\alpha, \beta)}(x, y) \\
\sum_{r=0}^{N} \sum_{s=0}^{N} C_{r s} P_{0,1}^{(\alpha, \beta)}(x, y) P_{r, s}^{(\alpha, \beta)}(x, y) \\
\vdots \\
\sum_{r=0}^{N} \sum_{s=0}^{N} C_{r s} P_{N, N}^{(\alpha, \beta)}(x, y) P_{r, s}^{(\alpha, \beta)}(x, y)
\end{array}\right] .
$$

Consider the $(p+1)$ th row of above vector. One puts

$$
P_{i, j}^{(\alpha, \beta)}(x, y) P_{r, s}^{(\alpha, \beta)}(x, y)=\sum_{k=0}^{N} \sum_{l=0}^{N} u_{k l} P_{k, l}^{(\alpha, \beta)}(x, y),
$$

Multiplying both sides of $(3.9)$ by $P_{m, n}^{(\alpha, \beta)}(x, y), m, n=0,1, \ldots, N$, and integrating the result from 0 to 1 yields:

$$
\begin{gathered}
\int_{0}^{1} \int_{0}^{1} P_{i, j}^{(\alpha, \beta)}(x, y) P_{r, s}^{(\alpha, \beta)}(x, y) P_{m, n}^{(\alpha, \beta)}(x, y) w^{(\alpha, \beta)}(x, y) d x d y= \\
\sum_{k=0}^{N} \sum_{l=0}^{N} u_{k l} \int_{0}^{1} \int_{0}^{1} P_{k, l}^{(\alpha, \beta)}(x, y) P_{m, n}^{(\alpha, \beta)}(x, y) w^{(\alpha, \beta)}(x, y) d x d y \\
=u_{m n} \theta_{m} \theta_{n} .
\end{gathered}
$$

Therefore,

$$
\begin{aligned}
u_{m n} & =\frac{1}{\theta_{m} \theta_{n}} \int_{0}^{1} P_{i}^{(\alpha, \beta)}(x) P_{r}^{(\alpha, \beta)}(x) P_{m}^{(\alpha, \beta)}(x) w^{(\alpha, \beta)}(x) d x \\
& \times \int_{0}^{1} P_{j}^{(\alpha, \beta)}(y) P_{s}^{(\alpha, \beta)}(y) P_{n}^{(\alpha, \beta)}(y) w^{(\alpha, \beta)}(y) d y .
\end{aligned}
$$

Now suppose $\int_{0}^{1} P_{i}^{(\alpha, \beta)}(x) P_{r}^{(\alpha, \beta)}(x) P_{m}^{(\alpha, \beta)}(x) w^{(\alpha, \beta)}(x) d x=q_{i r m}$. one gets

$$
u_{m n}=\frac{q_{i r m} q_{j s n}}{\theta_{m} \theta_{n}} .
$$

Substituting $u_{m n}$ into (3.9) one has:

$$
P_{i, j}^{(\alpha, \beta)}(x, y) P_{r, s}^{(\alpha, \beta)}(x, y)=\sum_{k=0}^{N} \sum_{l=0}^{N} \frac{q_{i r m} q_{j s n}}{\theta_{m} \theta_{n}} P_{k, l}^{(\alpha, \beta)}(x, y)
$$

Hence each component in the left side of relation (3.8) will be as follows:

$$
\begin{gathered}
\sum_{k=0}^{N} \sum_{l=0}^{N}\left\{\sum_{r=0}^{N} \sum_{s=0}^{N} \frac{C_{r s} q_{m r k} q_{n s l}}{\theta_{k} \theta_{l}}\right\} P_{k, l}^{(\alpha, \beta)}(x, y) \\
=\sum_{k=0}^{N} \sum_{l=0}^{N} \tilde{C}_{m(N+1)+n, k(N+1)+l} P_{k, l}^{(\alpha, \beta)}(x, y), \\
m, n=0,1, . ., N .
\end{gathered}
$$

Thus, the desirable result is obtained.

The Next theorem presents the general formula approximating the nonlinear term $v^{r}(x, y) u^{s}(x, y)$ which may appear in nonlinear equations. 
3.6. Theorem. If $c$ and $v$ are the $(N+1)^{2}$ vectors, $\tilde{c}$ and $\tilde{v}$ are the $(N+1)^{2} \times(N+1)$ operational matrices of the product such that

$$
\begin{aligned}
& u(x, y) \simeq \Phi^{T}(x, y) c=c^{T} \Phi(x, y), \quad v(x, y) \simeq \Phi^{T}(x, y) v=v^{T} \Phi(x, y), \\
& \Phi(x, y) \Phi^{T}(x, y) c \simeq \tilde{c} \Phi(x, y)
\end{aligned}
$$

and $\Phi(x, y) \Phi^{T}(x, y) v \simeq \tilde{v} \Phi(x, y)$, then the following proposition is hold:

$$
\begin{gathered}
v^{r}(x, y) u^{s}(x, y) \simeq v^{T}(\tilde{v})^{r-1} \tilde{B}_{s-1} \Phi(x, y), \quad B_{s-1}=\left(\tilde{c}^{T}\right)^{s-1} c, \\
r, s=1,2, \ldots .
\end{gathered}
$$

Proof. One has

$$
u^{2}(x, y) \simeq\left(\Phi^{T}(x, y) c\right)^{2}=c^{T} \Phi(x, y) \Phi^{T}(x, y) c \simeq c^{T} \tilde{c} \Phi(x, y),
$$

So, by use of induction, $u^{s}(x, y)$ will be approximated as

$$
u^{s}(x, y) \simeq c^{T}(\tilde{c})^{s-1} \Phi(x, y), \quad s=1,2, \ldots .
$$

To similar way, $v^{r}(x, y)$ is approximated as

$$
v^{r}(x, y) \simeq v^{T}(\tilde{v})^{r-1} \Phi(x, y), \quad r=1,2, \ldots .
$$

By using the expressed relations and induction is easily seen,

$$
\begin{gathered}
v^{r}(x, y) u^{s}(x, y) \simeq v^{T}(\tilde{v})^{r-1} \tilde{B}_{s-1} \Phi(x, y), \quad B_{s-1}=\left(\tilde{c}^{T}\right)^{s-1} c, \\
r, s=1,2, \ldots .
\end{gathered}
$$

\section{Best approximation and Convergence analysis}

In this section, the theorems on existence and uniqueness of best approximation, convergence analysis and error estimation of the proposed method are provided. For this reason, first the space $\mathbb{P}^{M}$ is considered as follows:

4.1. Definition. The set of all the linear combinations of $R_{0}^{(\alpha, \beta)}(x, y), R_{1}^{(\alpha, \beta)}(x, y)$, $\ldots, R_{M}^{(\alpha, \beta)}(x, y)$, which $M=(N+1)^{2}-1$, is represented by $\mathbb{P}^{M}$. On the other hand,

$$
\mathbb{P}^{M}=\operatorname{span}\left\{R_{0}^{(\alpha, \beta)}(x, y), R_{1}^{(\alpha, \beta)}(x, y), \ldots, R_{M}^{(\alpha, \beta)}(x, y)\right\}
$$

where two variables orthonormal polynomials $R_{i}^{(\alpha, \beta)}(x, y)$ are introduced by (3.6).

The following lemma is useful to prove the convexity and completeness properties of space $\mathbb{P}^{M}$.

4.2. Lemma. There is a number $\eta>0$ such that for every choice of scalars $\alpha_{0}, \alpha_{1}, \ldots, \alpha_{M}$ one has

$$
\begin{gathered}
\left\|\alpha_{0} R_{0}^{(\alpha, \beta)}(x, y)+\alpha_{1} R_{1}^{(\alpha, \beta)}(x, y)+\ldots+\alpha_{M} R_{M}^{(\alpha, \beta)}(x, y)\right\| \geq \\
\eta\left(\left|\alpha_{0}\right|+\left|\alpha_{1}\right|+\ldots+\left|\alpha_{M}\right|\right) .
\end{gathered}
$$

Proof. See [24].

4.3. Theorem. The space $\mathbb{P}^{M}$, defined by (4.1), is convex and complete. 
Proof. Suppose $v_{1}(x, y)$ and $v_{2}(x, y) \in \mathbb{P}^{M}$. One has for $0<\lambda<1$

$$
\lambda v_{1}(x, y)+(1-\lambda) v_{2}(x, y)=\sum_{i=0}^{M}\left(\lambda \omega_{i}^{1}+(1-\lambda) \omega_{i}^{2}\right) R_{i}^{(\alpha, \beta)}(x, y) \in \mathbb{P}^{M} .
$$

This shows that $\mathbb{P}^{M}$ is convex.

For proving the completeness property, let us consider Cauchy sequence

$$
\left\{w_{n}(x, y)\right\}_{n=0}^{\infty} \in \mathbb{P}^{M} .
$$

Then each $w_{n}(x, y)$ is a unique representation of the form

$$
w_{n}(x, y)=\sum_{i=0}^{M} \lambda_{i}^{(n)} R_{i}^{(\alpha, \beta)}(x, y)
$$

Since $\left\{w_{n}(x, y)\right\}_{n=0}^{\infty}$ is a Cauchy sequence, for every $\varepsilon>0$ there is a $N^{\prime}$ such that $\left\|w_{m}(x, y)-w_{n}(x, y)\right\|<\varepsilon$ where $m, n>N^{\prime}$. From this and Lemma 4.2, one has for $\eta>0$

$\varepsilon>\left\|w_{m}(x, y)-w_{n}(x, y)\right\|=\left\|\sum_{i=0}^{M}\left(\lambda_{i}^{(m)}-\lambda_{i}^{(n)}\right) R_{i}^{(\alpha, \beta)}(x, y)\right\| \geq \eta \sum_{i=0}^{M}\left|\lambda_{i}^{(m)}-\lambda_{i}^{(n)}\right|$.

Division by $\eta>0$ gives

$$
\left|\lambda_{i}^{(m)}-\lambda_{i}^{(n)}\right| \leq \sum_{i=0}^{M}\left|\lambda_{i}^{(m)}-\lambda_{i}^{(n)}\right|<\frac{\varepsilon}{\eta}
$$

This shows that each of the $M+1$ sequences $\left\{\lambda_{i}^{(n)}\right\}_{n=0}^{\infty}, i=0,1, \ldots, M$, is Cauchy in $\mathbb{R}$. Hence it converges. Let $\lambda_{i}$ denotes the limit. Using this $M+1$ limits $\lambda_{0}, \lambda_{1}, \ldots, \lambda_{M}$, one defines

$$
\bar{w}(x, y)=\sum_{i=0}^{M} \lambda_{i} R_{i}^{(\alpha, \beta)}(x, y)
$$

Clearly, $\bar{w}(x, y) \in \mathbb{P}^{M}$. Furthermore,

$$
\begin{aligned}
\left\|w_{n}(x, y)-\bar{w}(x, y)\right\| & =\left\|\sum_{i=0}^{M}\left(\lambda_{i}^{(n)}-\lambda_{i}\right) R_{i}^{(\alpha, \beta)}(x, y)\right\| \\
& \leq \sum_{i=0}^{M}\left|\lambda_{i}^{(n)}-\lambda_{i}\right|\left\|R_{i}^{(\alpha, \beta)}(x, y)\right\| .
\end{aligned}
$$

On the right, $\lambda_{i}^{(n)} \rightarrow \lambda_{i}$. Hence $\left\|w_{n}(x, y)-\bar{w}(x, y)\right\| \rightarrow 0$, that is, $w_{n}(x, y) \rightarrow \bar{w}(x, y)$. This shows that $\left\{w_{n}(x, y)\right\}_{n=0}^{\infty}$ is convergent in $\mathbb{P}^{M}$, and the completeness of $\mathbb{P}^{M}$ is proven.

4.4. Theorem. For every given continuous function $u(x, y)$ there exists a unique $u_{M}(x, y) \in$ $\mathbb{P}^{M}$ such that

$$
\delta=\inf _{\tilde{u} \in \mathbb{P}^{M}}\|u(x, y)-\tilde{u}(x, y)\|=\left\|u(x, y)-u_{M}(x, y)\right\| .
$$

Proof. Existence. By the definition of an infimum, there is a sequence $\left\{w_{n}(x, y)\right\}_{n=0}^{\infty}$ in $\mathbb{P}^{M}$ such that $\delta_{n} \rightarrow \delta$ where $\delta_{n}=\left\|u(x, y)-w_{n}(x, y)\right\|$. We show that $\left\{w_{n}(x, y)\right\}_{n=0}^{\infty}$ is Cauchy. Writing $v_{n}(x, y)=u(x, y)-w_{n}(x, y)$, one has $\left\|v_{n}(x, y)\right\|=\delta_{n}$ and

$$
\left\|v_{m}(x, y)+v_{n}(x, y)\right\|=2\left\|\frac{1}{2}\left(w_{m}(x, y)+w_{n}(x, y)\right)-u(x, y)\right\| \geq 2 \delta,
$$


because $\mathbb{P}^{M}$ is convex, so that $\frac{1}{2}\left(w_{m}(x, y)+w_{n}(x, y)\right) \in \mathbb{P}^{M}$. Furthermore, one has $v_{m}(x, y)-v_{n}(x, y)=w_{n}(x, y)-w_{m}(x, y)$. Hence by the parallelogram equality

$$
\begin{aligned}
\left\|w_{n}(x, y)-w_{m}(x, y)\right\|^{2} & =\left\|v_{m}(x, y)-v_{n}(x, y)\right\|^{2} \\
& =-\left\|v_{m}(x, y)+v_{n}(x, y)\right\|^{2} \\
& +2\left(\left\|v_{m}(x, y)\right\|^{2}+\left\|v_{n}(x, y)\right\|^{2}\right) \\
& \leq-(2 \delta)^{2}+2\left(\delta_{m}^{2}+\delta_{n}^{2}\right)<\varepsilon^{2} .
\end{aligned}
$$

This implies that $\left\{w_{n}(x, y)\right\}_{n=0}^{\infty}$ is Cauchy. Since $\mathbb{P}^{M}$ is complete, $\left\{w_{n}(x, y)\right\}_{n=0}^{\infty}$ converges, say, $w_{n}(x, y) \rightarrow \bar{w}(x, y) \in \mathbb{P}^{M}$. Since $\bar{w}(x, y) \in \mathbb{P}^{M}$, one has $\| u(x, y)-$ $\bar{w}(x, y) \| \geq \delta$. Also,

$$
\begin{aligned}
\|u(x, y)-\bar{w}(x, y)\| & \leq\left\|u(x, y)-w_{n}(x, y)\right\|+\left\|w_{n}(x, y)-\bar{w}(x, y)\right\| \\
& =\delta_{n}+\left\|w_{n}(x, y)-\bar{w}(x, y)\right\| \rightarrow \delta .
\end{aligned}
$$

This shows that $\|u(x, y)-\bar{w}(x, y)\|=\delta$.

Uniqueness. Let us assume that $\bar{w}(x, y) \in \mathbb{P}^{M}$ and $w_{0}(x, y) \in \mathbb{P}^{M}$ both satisfy

$$
\|u(x, y)-\bar{w}(x, y)\|=\delta, \quad\left\|u(x, y)-w_{0}(x, y)\right\|=\delta .
$$

By the parallelogram equality,

$$
\begin{aligned}
\left\|\bar{w}(x, y)-w_{0}(x, y)\right\|^{2} & =\left\|(\bar{w}(x, y)-u(x, y))-\left(w_{0}(x, y)-u(x, y)\right)\right\|^{2} \\
& =2\|\bar{w}(x, y)-u(x, y)\|^{2}+2\left\|w_{0}(x, y)-u(x, y)\right\|^{2} \\
& -\| \bar{w}(x, y)-u(x, y))+\left(w_{0}(x, y)-u(x, y)\right) \|^{2} \\
& =4 \delta^{2}-4\left\|\frac{1}{2}\left(\bar{w}(x, y)+w_{0}(x, y)\right)-u(x, y)\right\|^{2} \leq 0,
\end{aligned}
$$

because $\frac{1}{2}\left(\bar{w}(x, y)+w_{0}(x, y)\right) \in \mathbb{P}^{M}$. So that $\bar{w}(x, y)=w_{0}(x, y)$.

Orthogonality. We assume there be a $0 \neq w_{1}(x, y) \in \mathbb{P}^{M}$ such that

$$
\left(z(x, y), w_{1}(x, y)\right)=\gamma \neq 0
$$

where $z(x, y)=u(x, y)-\bar{w}(x, y)$ and $(.,$.$) denotes the inner product. Furthermore, for$ any scalar $\eta$,

$$
\begin{aligned}
\left\|z(x, y)-\eta w_{1}(x, y)\right\|^{2} & =\left(z(x, y)-\eta w_{1}(x, y), z(x, y)-\eta w_{1}(x, y)\right) \\
& =\|z(x, y)\|^{2}-\bar{\eta} \gamma-\eta\left(\bar{\gamma}-\bar{\eta}\left\|w_{1}(x, y)\right\|^{2}\right),
\end{aligned}
$$

Choosing $\bar{\eta}=\frac{\bar{\gamma}}{\left\|w_{1}(x, y)\right\|^{2}}$ yields

$$
\left\|z(x, y)-\eta w_{1}(x, y)\right\|^{2}=\|z(x, y)\|^{2}-\frac{\bar{\gamma}}{\left\|w_{1}(x, y)\right\|^{2}}=\delta^{2}-\frac{\bar{\gamma}}{\left\|w_{1}(x, y)\right\|^{2}} \geq \delta^{2},
$$

But this is impossible because one has $\left\|z(x, y)-\eta w_{1}(x, y)\right\| \geq \delta$ by the definition of $\delta$. Hence the assumption can not be hold. So $(z(x, y), \tilde{u}(x, y))=0, \forall \tilde{u}(x, y) \in \mathbb{P}^{M}$.

Now it is shown that $\bar{w}(x, y)=u_{M}(x, y)$. It was proven that $\bar{w}(x, y)$ is the best approximation for $u(x, y)$. So,

$$
\forall j, j=0,1, . ., M, \quad\left(u(x, y)-\bar{w}(x, y), R_{j}(x, y)\right)=0,
$$

where two variables polynomials $R_{i}^{(\alpha, \beta)}(x, y)$ are introduced by (3.6). One has from this point,

$$
\begin{aligned}
\left(\bar{w}(x, y)-u_{M}(x, y), R_{j}(x, y)\right) & =\left(u(x, y)-(u(x, y)-\bar{w}(x, y))-u_{M}(x, y), R_{j}(x, y)\right) \\
& =\left(u(x, y), R_{j}(x, y)\right)-\left(u(x, y)-\bar{w}(x, y), R_{j}(x, y)\right) \\
& -\left(u_{M}(x, y), R_{j}(x, y)\right) \\
& =\omega_{j}-\omega_{j}=0, \quad j=0,1, \ldots, M
\end{aligned}
$$


Therefore $\bar{w}(x, y)-u_{M}(x, y)=0$. This shows that $u_{M}(x, y)=\bar{w}(x, y)$ and proof is completed.

Two following theorems state the decaying of the Jacobi coefficients and the convergence of the best approximation.

4.5. Theorem. The Jacobi coefficients $\omega_{i}$, introduced by (3.6), decay when the number of the terms of the partial sum of the series solution, $N$, increases.

Proof. Employing the $\omega_{i}$ and the properties of the orthogonality of $R_{i}(x, y)$, we have

$$
\begin{aligned}
\int_{0}^{1} \int_{0}^{1} S_{M}(x, y) & u(x, y) w^{(\alpha, \beta)}(x, y) d x d y= \\
& \sum_{i=0}^{M} \omega_{i} \int_{0}^{1} \int_{0}^{1} R_{i}(x, y) u(x, y) w^{(\alpha, \beta)}(x, y) d x d y \\
& =\sum_{i=0}^{M} \omega_{i}^{2}
\end{aligned}
$$

If $u^{2}(x, y) w^{(\alpha, \beta)}(x, y)$ as well as $u(x, y) w^{(\alpha, \beta)}(x, y)$ is integrable, then

$$
\begin{aligned}
\int_{0}^{1} \int_{0}^{1}[u(x, y)- & \left.S_{M}(x, y)\right]^{2} w^{(\alpha, \beta)}(x, y) d x d y= \\
& \int_{0}^{1} \int_{0}^{1} u^{2}(x, y) w^{(\alpha, \beta)}(x, y) d x d y \\
& -2 \int_{0}^{1} \int_{0}^{1} u(x, y) S_{M}(x, y) w^{(\alpha, \beta)}(x, y) d x d y \\
& +\int_{0}^{1} \int_{0}^{1} S_{M}^{2}(x, y) w^{(\alpha, \beta)}(x, y) d x d y \\
& =\int_{0}^{1} \int_{0}^{1} u^{2}(x, y) w^{(\alpha, \beta)}(x, y) d x d y-\sum_{i=0}^{M} \omega_{i}^{2}
\end{aligned}
$$

Therefore,

$$
\sum_{i=0}^{M} \omega_{i}^{2} \leq \int_{0}^{1} \int_{0}^{1} u^{2}(x, y) w^{(\alpha, \beta)}(x, y) d x d y, \quad \forall N \in \mathbb{N}
$$

Consequently $\sum_{i=0}^{\infty} \omega_{i}^{2}$ is convergent and $\lim _{i \rightarrow \infty} \omega_{i}=0$.

Theorem 4.5 states the given function $u(x, y)$ may be approximated using only the finite numbers of two variables Jacobi polynomials.

4.6. Theorem. The series solution (3.3) converges towards $u(x, y)$ in (3.2).

Proof. Consider the relation (3.6) and define the sequence partial sums

$$
\left\{S_{M}(x, y)\right\}_{M=0}^{\infty}
$$

as follows,

$$
S_{M}(x, y)=\sum_{i=0}^{M} \omega_{i} R_{i}(x, y)
$$


Let suppose that the $S_{M}(x, y)$ and $S_{L}(x, y)$ are partial sums with $M>L$. It is going to prove that $\left\{S_{M}(x, y)\right\}_{M=0}^{\infty}$ is a Cauchy sequence in $\mathbb{P}^{M}$. For this purpose, it is worked out as follows:

$$
\begin{aligned}
\left\|\sum_{i=L+1}^{M} \omega_{i} R_{i}(x, y)\right\|^{2} & =\left(\sum_{i=L+1}^{M} \omega_{i} R_{i}(x, y), \sum_{j=L+1}^{M} \omega_{j} R_{j}(x, y)\right) \\
& =\sum_{i=L+1}^{M} \sum_{j=L+1}^{M} \omega_{i} \bar{\omega}_{j}\left(R_{i}(x, y), R_{j}(x, y)\right) \\
& =\sum_{i=L+1}^{M}\left|\omega_{i}\right|^{2} .
\end{aligned}
$$

That is $\left\|S_{M}(x, y)-S_{L}(x, y)\right\|^{2}=\sum_{i=L+1}^{M}\left|\omega_{i}\right|^{2}$. From Bessel inequality $\sum_{i=0}^{\infty}\left|\omega_{i}\right|^{2}$ is convergent and hence $\left\|S_{M}(x, y)-S_{L}(x, y)\right\|^{2} \leq \varepsilon^{2}$. This shows $\left\{S_{M}(x, y)\right\}_{M=0}^{\infty}$ is a Cauchy sequence. Since $\mathbb{P}^{M}$ is complete one has $S_{M}(x, y) \rightarrow S(x, y) \in \mathbb{P}^{M}$. We show $S(x, y)=u(x, y)$ :

$$
\begin{aligned}
\left(S(x, y)-u(x, y), R_{j}(x, y)\right) & =\left(S(x, y), R_{j}(x, y)\right)-\left(u(x, y), R_{j}(x, y)\right) \\
& =\left(\lim _{M \rightarrow \infty} S_{M}(x, y), R_{j}(x, y)\right)-\left(u(x, y), R_{j}(x, y)\right) \\
& =\lim _{M \rightarrow \infty}\left(S_{M}(x, y), R_{j}(x, y)\right)-\left(u(x, y), R_{j}(x, y)\right) \\
& =\omega_{j}-\omega_{j}=0, \\
& \Rightarrow S(x, y)-u(x, y)=0, \quad j=0,1, \ldots, M .
\end{aligned}
$$

Hence,

$$
S(x, y)=\sum_{i=0}^{\infty} \omega_{i} R_{i}(x, y)=\sum_{i=0}^{\infty} \sum_{j=0}^{\infty} C_{i j} P_{i}^{(\alpha, \beta)}(x) P_{j}^{(\alpha, \beta)}(y)=u(x, y)
$$

Whenever the solution of a problem is not known, specially in nonlinear phenomena, an error estimator is needed as an essential component of the computational algorithm. To this end, an error estimator for the proposed method is presented in this section.

For simplicity, discussed equations are written in the operator form

$$
\mathcal{D} u(x, y)=f(x, y)
$$

where $\mathcal{D}$ is a integro-partial differential operator. Define the error function as

$$
e_{N}(x, y)=u(x, y)-u_{N}(x, y) .
$$

Substituting $u_{N}(x, y)$ into given equations yields

$$
\mathcal{D} u_{N}(x, y)=f(x, y)+H_{N}(x, y)
$$

where $H_{N}(x, y)$ is a perturbation term. Subtracting (4.3) from (4.2) gives

$$
\mathcal{D} e_{N}(x, y)=-H_{N}(x, y) \text {. }
$$

Now, it can be proceeded by the same way as the basic problem is solved to get the estimation $e_{N, M}(x, y)$ to the error function $e_{N}(x, y)$. Note the stated approximations are also substituted in the conditions of the given problem. Subsequently, the conditions of new problem will be homogeneous. 


\section{Numerical results}

In this section, four examples are given to certify the efficiency and accuracy of the proposed method where the maximum absolute and estimate errors are reported for different values of parameters $\alpha$ and $\beta$. Also, the absolute and estimate errors are computed at some arbitrary selected points.

5.1. Example. Consider the following linear Volterra-Fredholm integro-partial differential equation.

$$
u_{x x}(x, y)+\sin (x y) u(x, y)=x y \sin (x y)-\frac{1}{6} y^{3}+\int_{0}^{y} \int_{0}^{1} t s u_{t}(t, s) d t d s,
$$

with the following conditions,

$$
u_{x}(0, y)=y, \quad u(0, y)=0 .
$$

The exact solution of this problem is $u(x, y)=x y$. First, let us consider the following approximation,

$$
u_{x x}(x, y) \simeq \Phi^{T}(x, y) C .
$$

Integrating (5.3) with respect to $x$ from 0 to $x$, one gets the following approximation for $u_{x}(x, y)$.

$$
u_{x}(x, y) \simeq \Phi^{T}(x, y) P_{x}^{T} C+u_{x}(0, y) \simeq \Phi^{T}(x, y) P_{x}^{T} C+\Phi^{T}(x, y) V,
$$

where $u_{x}(0, y)$ is approximated by $\Phi^{T}(x, y) V$ which $V$ is a $(N+1) \times 1$ known vector. Again, integrating (5.4) with respect to $x$ from 0 to $x$, one obtains the following approximation for $u(x, y)$,

$$
u(x, y) \simeq \Phi^{T}(x, y)\left(P_{x}^{T}\right)^{2} C+\Phi^{T}(x, y) P_{x}^{T} V .
$$

In order to approximate the integral part in the (5.1), the kernel ts is approximated as follows:

$$
t s \simeq \Phi^{T}(x, y) K \Phi(t, s),
$$

where $K$ is a $(N+1)^{2} \times(N+1)^{2}$ known matrix and is determined by inner product. Now, the integral part in (5.1) is approximated as:

$$
\begin{aligned}
\int_{0}^{y} \int_{0}^{1} t s u_{t}(t, s) d t d s & \simeq \int_{0}^{y} \int_{0}^{1} \Phi^{T}(x, y) K \Phi(t, s)\left\{\Phi^{T}(t, s) P_{x}^{T} C\right. \\
& \left.+\Phi^{T}(t, s) V\right\} d t d s \\
& \simeq \Phi^{T}(x, y) K\{\tilde{V}+\tilde{B}\} P_{y} A
\end{aligned}
$$

where $\tilde{V}$ is operational matrix of product and its entries are determined in terms of the components of the vector $V, \tilde{B}$ is operational matrix of product corresponding to vector $B=P_{x}^{T} C$, and $A=\int_{0}^{1} \Phi(t, y) d t$. Substituting the approximations (5.3)-(5.7) into (5.1), leads to the following linear algebraic equation.

$$
\begin{aligned}
\Phi^{T}(x, y) C & +\sin (x y) \Phi^{T}(x, y)\left\{\left(P_{x}^{T}\right)^{2} C+P_{x}^{T} V\right\} \\
& -\Phi^{T}(x, y) K\{\tilde{V}+\tilde{B}\} P_{y} A-x y \sin (x y)+\frac{1}{6} y^{3}=0 .
\end{aligned}
$$

Setting $N=3$ and using the roots of $P_{4}^{(\alpha, \beta)}(x)$ and $P_{4}^{(\alpha, \beta)}(y)$ in the $x$ and $y$-directions, (5.8) is collocated in 16 inner tensor points for different values of parameters $\alpha$ and $\beta$. Hereby, the (5.8) reduces the problem to solve a system of linear algebraic equations and unknown coefficients are obtained for some values of parameters $\alpha$ and $\beta$. Table 1 shows 
the maximum absolute and estimate errors of the approximate solutions for different values of $\alpha$ and $\beta$. Table 2 displays different values of the exact and approximate solutions in points $(x, y)=(0.1 i, 0.1 i),(i=1,2, \ldots, 10)$ for $\alpha=\beta=-1 / 4$. As can be seen from Tables the results of the solutions obtained by Jacobi polynomials method are almost the same as the results of the exact solutions.

Table 1. Maximum absolute and estimate errors of Example 5.1 for different values $\alpha$ and $\beta$
\begin{tabular}{|c||c|c||c|c|c|}
\hline$(\alpha, \beta)$ & Error $_{A b s}$ & Error $_{\text {Est }}$ & $(\alpha, \beta)$ & Error $A b s_{1}$ & Error $_{\text {Est }}$ \\
\hline \hline$(0,0)$ & $5.2715 \times 10^{-21}$ & $4.7132 \times 10^{-21}$ & $\left(\frac{1}{4}, \frac{1}{4}\right)$ & $4.9562 \times 10^{-20}$ & $3.8921 \times 10^{-21}$ \\
\hline$(1,1)$ & $2.4802 \times 10^{-20}$ & $2.2000 \times 10^{-20}$ & $\left(-\frac{1}{4},-\frac{1}{4}\right)$ & $5.0940 \times 10^{-19}$ & $2.2476 \times 10^{-19}$ \\
\hline$(2,2)$ & $5.3375 \times 10^{-20}$ & $3.8880 \times 10^{-21}$ & $\left(\frac{3}{4}, \frac{3}{4}\right)$ & $9.8549 \times 10^{-19}$ & $4.8000 \times 10^{-19}$ \\
\hline$\left(\frac{1}{2}, \frac{1}{2}\right)$ & $1.5140 \times 10^{-21}$ & $1.5829 \times 10^{-21}$ & $\left(\frac{1}{10}, \frac{1}{10}\right)$ & $1.3500 \times 10^{-19}$ & $3.8930 \times 10^{-21}$ \\
\hline
\end{tabular}

Table 2. Maximum absolute and estimate errors of Example 5.1 for various values of $\alpha=\beta=-\frac{1}{4}$

\begin{tabular}{|c||c|c|c|c|}
\hline$\left(x_{i}, y_{i}\right)$ & Exact value & Approximate value & Error $A$ bs & Error sst $^{-21}$ \\
\hline \hline$(0.1,0.1)$ & 0.01 & 0.0099999999999999999918 & $8.20 \times 10^{-21}$ & $1.6649 \times 10^{-21}$ \\
\hline$(0.2,0.2$ & 0.04 & 0.040000000000000000004 & $4.00 \times 10^{-21}$ & $4.7592 \times 10^{-21}$ \\
\hline$(0.3,0.3)$ & 0.09 & 0.090000000000000000008 & $8.00 \times 10^{-21}$ & $6.7477 \times 10^{-21}$ \\
\hline$(0.4,0.4)$ & 0.16 & 0.16000000000000000000 & $8.00 \times 10^{-21}$ & $5.2854 \times 10^{-21}$ \\
\hline$(0.5,0.5)$ & 0.25 & 0.25000000000000000001 & $1.00 \times 10^{-20}$ & $61.9554 \times 10^{-21}$ \\
\hline$(0.6,0.6)$ & 0.36 & 0.35999999999999999999 & $1.00 \times 10^{-20}$ & $1.7448 \times 10^{-20}$ \\
\hline$(0.7,0.7)$ & 0.49 & 0.49000000000000000000 & 0.00 & $04.3963 \times 10^{-20}$ \\
\hline$(0.8,0.8)$ & 0.64 & 0.64000000000000000000 & 0.00 & $8.4713 \times 10^{-20}$ \\
\hline$(0.9,0.9)$ & 0.84 & 0.81000000000000000002 & $2.00 \times 10^{-20}$ & $1.4349 \times 10^{-19}$ \\
\hline$(1,1)$ & 1.00 & 1.0000000000000000001 & $1.00 \times 10^{-19}$ & $2.2476 \times 10^{-19}$ \\
\hline
\end{tabular}

5.2. Example. Consider the following linear Volterra integro-partial differential equation.

$$
u_{x}(x, y)+u_{y}(x, y)=-1+\exp (x)+\exp (y)+\exp (x+y)+\int_{0}^{x} \int_{0}^{y} u(t, s) d s d t,
$$

with the conditions $u(x, 0)=\exp (x)$ and $u(0, y)=\exp (y)$. The exact solution of this problem is $u(x, y)=\exp (x+y)$. Let us consider the following approximation,

$$
u_{x y}(x, y) \simeq \Phi^{T}(x, y) C .
$$

Integrating (5.10) with respect to $y$ from 0 to $y$, one obtains the following approximation for $u_{x}(x, y)$.

$$
u_{x}(x, y) \simeq \Phi^{T}(x, y) P_{y}^{T} C+u_{x}(x, 0) \simeq \Phi^{T}(x, y) P_{y}^{T} C+\Phi^{T}(x, y) V_{1} .
$$

Now, integrating (5.10) with respect to $x$ from 0 to $x$, one gets the following approximation for $u_{y}(x, y)$ as follows:

$$
u_{y}(x, y) \simeq \Phi^{T}(x, y) P_{x}^{T} C+u_{y}(0, y) \simeq \Phi^{T}(x, y) P_{x}^{T} C+\Phi^{T}(x, y) V_{2} .
$$

Also, by integrating the relation (5.11) with respect to $x$ from 0 to $x$ an approximation yields for $u(x, y)$ as follows:

$$
\begin{aligned}
u(x, y) & \simeq \Phi^{T}(x, y) P_{x}^{T} P_{y}^{T} C+\Phi^{T}(x, y) P_{x}^{T} V_{1}+u(0, y) \\
& \simeq \Phi^{T}(x, y) P_{x}^{T} P_{y}^{T} C+\Phi^{T}(x, y) P_{x}^{T} V_{1}+\Phi^{T}(x, y) V_{1} .
\end{aligned}
$$

The kernel is approximated as follows:

$$
1 \simeq \Phi^{T}(x, y) K \Phi(t, s) .
$$


Now, the integral part in $(5.9)$ is approximated as:

$$
\begin{aligned}
\int_{0}^{x} \int_{0}^{y} u(t, s) d t d s & \simeq \int_{0}^{y} \int_{0}^{1} \Phi^{T}(x, y) K \Phi(t, s) \Phi^{T}(x, y)\left\{P_{x}^{T} P_{y}^{T} C\right. \\
& \left.+P_{x}^{T} V_{1}+V_{1}\right\} d s d t \\
& \simeq \Phi^{T}(x, y) K \tilde{A} P_{y} P_{x} \Phi(x, y)
\end{aligned}
$$

where $\tilde{A}$ is operational matrix of product corresponding to vector $A=P_{x}^{T} P_{y}^{T} C+P_{x}^{T} V_{1}+$ $V_{1}$. Substituting the approximations (5.11)-(5.15) into (5.9), leads to the following linear algebraic equation.

$$
\begin{aligned}
\Phi^{T}(x, y) P_{y}^{T} C & +\Phi^{T}(x, y) V_{1}+\Phi^{T}(x, y) P_{x}^{T} C+\Phi^{T}(x, y) V_{2} \\
& -\Phi^{T}(x, y) K \tilde{A} P_{y} P_{x} \Phi(x, y)+1-e^{x}-e^{y}-e^{x+y}=0 .
\end{aligned}
$$

Setting $N=7$ and using the roots of $P_{8}^{(\alpha, \beta)}(x)$ and $P_{8}^{(\alpha, \beta)}(y)$ in the $x$ and $y$-directions, (5.16) is collocated in 64 inner tensor points for different values of parameters $\alpha$ and $\beta$. Hereby, the (5.16) reduces the problem to solve a system of linear algebraic equations and unknown coefficients are obtained for some values of parameters $\alpha$ and $\beta$. Table 3 displays the maximum absolute and estimate errors of the approximate solutions for different values of $\alpha$ and $\beta$. Table 4 shows different values of the exact and approximate solutions in points $(x, y)=(0.2 i, 0.2 i),(i=1,2, \ldots, 5)$ for $\alpha=\beta=1$ and $N=4,7,8$. It can be observed from Table 4 that the errors decrease as $N$ increases. Also,

Table 3. Maximum absolute and estimate errors of Example 5.2 for $N=7$ and various values of $\alpha$ and $\beta$

\begin{tabular}{|c||c|c|}
\hline$(\alpha, \beta)$ & Error $_{A b s}$ & Error $_{\text {Est }}$ \\
\hline \hline$(0,0)$ & $1.7484 \times 10^{-8}$ & $8.5474 \times 10^{-9}$ \\
\hline$\left(\frac{1}{2}, \frac{1}{2}\right)$ & $3.1164 \times 10^{-8}$ & $1.4839 \times 10^{-8}$ \\
\hline$(1,1)$ & $4.6983 \times 10^{-8}$ & $2.0999 \times 10^{-8}$ \\
\hline$\left(-\frac{1}{4},-\frac{1}{4}\right)$ & $8.2454 \times 10^{-8}$ & $4.8751 \times 10^{-8}$ \\
\hline$\left(\frac{1}{4}, \frac{1}{4}\right)$ & $2.4007 \times 10^{-8}$ & $1.1603 \times 10^{-8}$ \\
\hline
\end{tabular}

Table 4. Comparison of the exact and approximate solutions of Example 5.2 for $N=4,7,8$ and $\alpha=\beta=1$

\begin{tabular}{|c||c|c|c|c|c|}
\hline$\left(x_{i}, y_{i}\right)$ & $u_{\text {Exact }}$ & Error $\left(u_{4}\right)$ & Error $\left(u_{7}\right)$ & $u_{8}(x, y)$ & Error $\left(u_{8}\right)$ \\
\hline \hline$(0.2,0.2)$ & 1.49182469764 & $2.7289 \times 10^{-5}$ & $1.6429 \times 10^{-9}$ & 1.49182469766 & $2.2390 \times 10^{-11}$ \\
\hline$(0.4,0.4)$ & 2.22554092849 & $3.3508 \times 10^{-5}$ & $4.1350 \times 10^{-10}$ & 2.22554092853 & $3.5908 \times 10^{-11}$ \\
\hline$(0.6,0.6)$ & 3.32011692274 & $3.2674 \times 10^{-5}$ & $3.5768 \times 10^{-10}$ & 3.32011692270 & $3.8762 \times 10^{-11}$ \\
\hline$(0.8,0.8)$ & 4.95303242440 & $5.6798 \times 10^{-5}$ & $3.0065 \times 10^{-9}$ & 4.95303242437 & $2.7025 \times 10^{-11}$ \\
\hline$(1,1)$ & 7.38905609893 & $5.8038 \times 10^{-4}$ & $4.6983 \times 10^{-8}$ & 7.38905609744 & $1.4893 \times 10^{-9}$ \\
\hline
\end{tabular}

5.3. Example. Consider the following nonlinear system of Fredholm integro-partial differential equation.

$$
\left\{\begin{array}{l}
u(x, y)-v(x, y)+\int_{0}^{1} u(t, y) v_{t}(t, y) d t=f_{1}(x, y) \\
v(x, y)+3 u(x, y)-\int_{0}^{1} u_{t}(t, y) v(t, y) d t=f_{2}(x, y)
\end{array}\right.
$$

where $f_{1}(x, y)=x^{2} \cos (y)-y \sin (x)-y \cos (y)(\sin (1)-2 \cos (1))$ and $f_{2}(x, y)=$ $y \sin (x)+3 x^{2} \cos (y)-2 y \cos (y)(\sin (1)-\cos (1))$ with boundary conditions $u(0, y)=$ 0 and $v(0, y)=0$. The exact solutions of this problem are $u(x, y)=x^{2} \cos (y)$ and 


$$
\begin{aligned}
& v(x, y)=y \sin (x) \text {. The following approximations are used for } N=5, \\
& u_{x}(x, y) \simeq \Phi^{T}(x, y) C_{1}, \quad u(x, y) \simeq \Phi^{T}(x, y) P_{x}^{T} C_{1} \quad v_{x}(x, y) \simeq \Phi^{T}(x, y) C_{2}, \\
& v(x, y) \simeq \Phi^{T}(x, y) P_{x}^{T} C_{2} \quad 1 \simeq \Phi^{T}(x, y) K \Phi(t, y), \\
& u(t, y) v_{t}(t, y) \simeq A^{T} \Phi(t, y) \Phi^{T}(t, y) C_{2} \simeq A^{T} \tilde{C}_{2} \Phi(t, y)=\Phi^{T}(t, y) \tilde{C}_{2}^{T} A, \\
& u_{t}(t, y) v(t, y) \simeq C_{1}^{T} \Phi(t, y) \Phi^{T}(t, y) B \simeq C_{1}^{T} \tilde{B} \Phi(t, y)=\Phi^{T}(t, y) \tilde{B}^{T} C_{1}, \\
& \int_{0}^{1} u(t, y) v_{t}(t, y) d t \simeq \Phi(x, y) K E \tilde{C}_{2}^{T} A, \\
& \int_{0}^{1} u_{t}(t, y) v(t, y) d t \simeq \Phi(x, y) K E \tilde{B}^{T} C_{1},
\end{aligned}
$$

where $A=P_{x}^{T} C_{1}, B=P_{x}^{T} C_{2}, \tilde{C}_{2}$ and $\tilde{B}$ are the operational matrices of product corresponding to the vectors $C_{2}$ and $B$, and $E$ is the following matrix:

$$
E=\int_{0}^{1} \Phi(t, y) \Phi^{T}(t, y) d t
$$

Note for approximating the nonlinear terms $u(t, y) v_{t}(t, y)$ and $u_{t}(t, y) v(t, y)$ has been used the Theorem 3.6. Substituting above approximations into system (5.17), leads to the following nonlinear system of algebraic equations.

$$
\left\{\begin{array}{l}
\Phi^{T}(x, y) P_{x}^{T} C_{1}-\Phi^{T}(x, y) P_{x}^{T} C_{2}+\Phi(x, y) K E \tilde{C}_{2}^{T} A=f_{1}(x, y), \\
\Phi^{T}(x, y) P_{x}^{T} C_{2}+3 \Phi^{T}(x, y) P_{x}^{T} C_{1}-\Phi(x, y) K E \tilde{B}^{T} C_{1}=f_{2}(x, y),
\end{array}\right.
$$

Setting $N=5$ and using the roots of $P_{6}^{(\alpha, \beta)}(x)$ and $P_{6}^{(\alpha, \beta)}(y)$ in the $x$ and $y$-directions,

\begin{tabular}{|c|c|c|c|c|c|c|}
\hline$\left(x_{i}, y_{i}\right)$ & $u_{\text {Exact }}$ & $u_{5}(x, y)$ & Error $_{A b s}$ & $v_{\text {Exact }}$ & $v_{5}(x, y)$ & Error $A b s$ \\
\hline$(0.1,0.1)$ & 0.00995004 & 0.00995002 & $2.0419 \times 10^{-8}$ & 0.00998334 & $0.00998326 e$ & $7.8601 \times 10^{-8}$ \\
\hline$(0.2,0.2)$ & $0.03920266 e$ & 0.03920263 & $2.6117 \times 10^{-8}$ & 0.03973387 & 0.03973376 & $1.0216 \times 10^{-7}$ \\
\hline$(0.3,0.3)$ & $0.08598028 e$ & 0.08598027 & $1.2031 \times 10^{-8}$ & 0.08865606 & 0.08865595 & $1.14 \times 10^{-7}$ \\
\hline$(0.4,0.4)$ & 0.14736975 & 0.14736970 & $5.6500 \times 10^{-8}$ & 0.15576734 & 0.15576711 & $2.2310 \times 10^{-7}$ \\
\hline$(0.5,0.5)$ & 0.21939564 & 0.21939551 & $1.2727 \times 10^{-7}$ & 0.23971277 & 0.23971243 & $3.3863 \times 10^{-7}$ \\
\hline$(0.6,0.6)$ & 0.29712082 & 0.29712075 & $7.1500 \times 10^{-8}$ & 0.33878548 & 0.33878517 & $3.1259 \times 10^{-7}$ \\
\hline$(0.7,0.7)$ & 0.37477267 & 0.37477277 & $9.8920 \times 10^{-8}$ & 0.45095238 & 0.45095218 & $2.0102 \times 10^{-7}$ \\
\hline$(0.8,0.8)$ & 0.44589229 & 0.445892296 & $2.2183 \times 10^{-9}$ & 0.57388487 & 0.57388456 & $3.1067 \times 10^{-7}$ \\
\hline$(0.9,0.9)$ & .50350407 & 0.50350365 & $4.1437 \times 10^{-7}$ & 0.70499422 & 0.70499360 & $6.1633 \times 10^{-7}$ \\
\hline$(1,1)$ & 0.54030231 & 0.54030426 & $1.9550 \times 10^{-6}$ & 0.84147098 & 0.84147191 & $9.2444 \times 10^{-7}$ \\
\hline
\end{tabular}
each equation of the system (5.18) is collocated in 36 inner tensor points for different values of parameters $\alpha$ and $\beta$. Hereby, the system (5.18) reduces the problem to solve a system of nonlinear algebraic equations and 72 unknown coefficients are obtained for some values of parameters $\alpha$ and $\beta$ by using Newton iterative method. Table 5 shows different values of the exact and approximate solutions in points $(x, y)=(0.1 i, 0.1 i),(i=$ $1,2, \ldots, 10)$ for $\alpha=\beta=\frac{1}{2}$. Also, in Figure 1 the exact and approximate solutions are compared for the case $\alpha=\beta=0$. Also, the absolute errors functions obtained by the proposed method are displayed in Figure 1 for $\alpha=\beta=0$.

5.4. Example. Consider the following nonlinear system Volterra integro-partial differential equation.

(5.19) $\left\{\begin{array}{l}u_{y}(x, y)+v(x, y)-\int_{0}^{y} \int_{0}^{x} t \sin (s)\left(u^{2}(t, s)-v^{2}(t, s)\right) d t d s=f_{1}(x, y) \\ u_{y}(x, y)+v_{y}(x, y)+u(x, y)-\int_{0}^{y} \int_{0}^{x} t \cos (s)\left(u(t, s)-v_{s}(t, s)\right) d t d s= \\ f_{2}(x, y),\end{array}\right.$ 
Figure 1. Comparison of the exact and approximate solutions and their error functions for $\alpha=\beta=0$ in Example 5.3: Plots of $(a) u_{5}(x, y)$, (b) $v_{5}(x, y),(c)$ error function of $u(x, y),(d)$ error function of $v(x, y)$

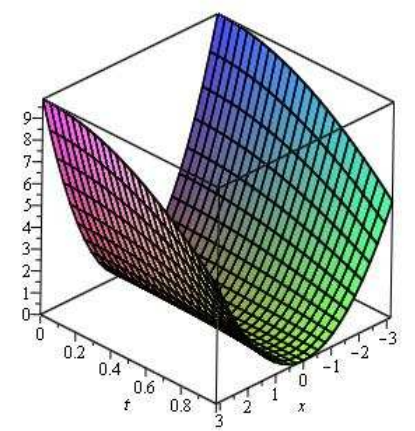

(a)

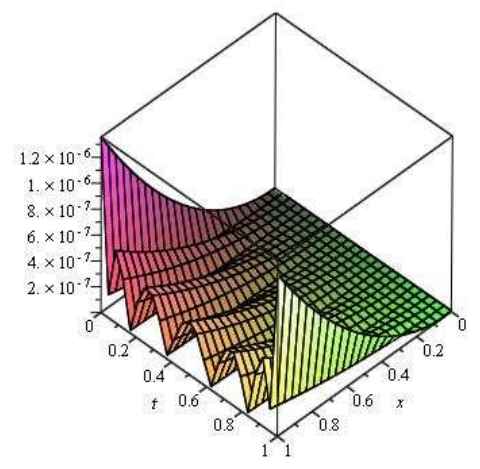

(c)

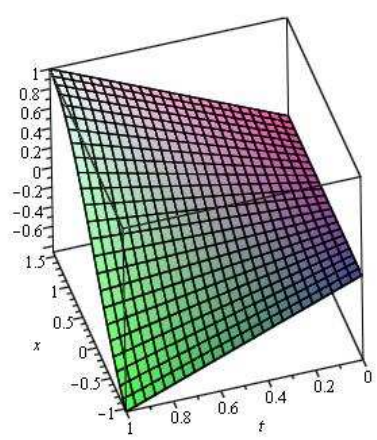

(b)

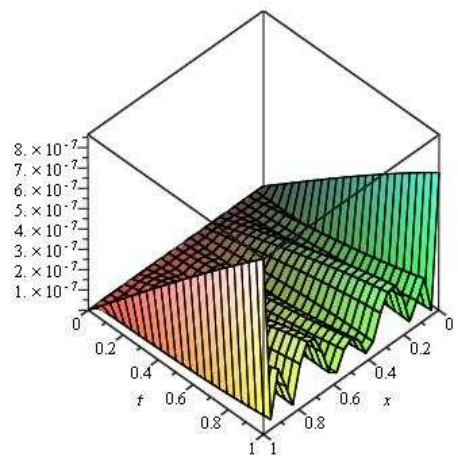

(d)

where $f_{1}(x, y)=\frac{1}{12}\left(1+2 \cos ^{3}(y)-3 \cos (y)\right) x^{4}$ and $f_{2}(x, y)=x(2 \cos (y)-\sin (y))$ with the conditions $u(x, 0)=x$ and $v(x, 0)=0$. The exact solutions of this problem are $u(x, y)=x \cos (y)$ and $v(x, y)=x \sin (y)$. The following approximations are used for $N=5$,

$u_{y}(x, y) \simeq \Phi^{T}(x, y) C_{1}, \quad v_{y}(x, y) \simeq \Phi^{T}(x, y) C_{2}$,

$u(x, y) \simeq \Phi^{T}(x, y) P_{y}^{T} C_{1}+u(x, 0) \simeq \Phi^{T}(x, y) P_{y}^{T} C_{1}+\Phi^{T}(x, y) V$,

$v(x, y) \simeq \Phi^{T}(x, y) P_{y}^{T} C_{2}, \quad t \sin (s) \simeq \Phi^{T}(x, y) K_{1} \Phi^{T}(t, s)$,

$t \cos (s) \simeq \Phi^{T}(x, y) K_{2} \Phi^{T}(t, s)$,

$u^{2}(x, y) \simeq A_{1}^{T} \Phi(x, y) \Phi^{T}(x, y) A_{1} \simeq A_{1}^{T} \tilde{A}_{1} \Phi(x, y)=\Phi^{T}(x, y) B_{1}$,

$v^{2}(x, y) \simeq A_{2}^{T} \Phi(x, y) \Phi^{T}(x, y) A_{2} \simeq A_{2}^{T} \tilde{A}_{2} \Phi(x, y)=\Phi^{T}(x, y) B_{2}$,

$\int_{0}^{y} \int_{0}^{x} t \sin (s)\left(u^{2}(t, s)-v^{2}(t, s)\right) d t d s \simeq \Phi^{T}(x, y) K_{1}\left(\tilde{B}_{1}-\tilde{B}_{2}\right) P_{x} P_{y} \Phi(x, y)$,

$\int_{0}^{y} \int_{0}^{x} t \cos (s)\left(u(t, s)-v_{s}(t, s)\right) d t d s \simeq \Phi^{T}(x, y) K_{2}\left(\tilde{A}_{1}-\tilde{C}_{2}\right) P_{x} P_{y} \Phi(x, y)$, 
Figure 2. Comparison of the exact and approximate solutions and their error functions for $\alpha=\beta=0$ in Example 5.4: Plots of $(a) u_{5}(x, y)$, (b) $v_{5}(x, y),(c)$ error function of $u(x, y),(d)$ error function of $v(x, y)$

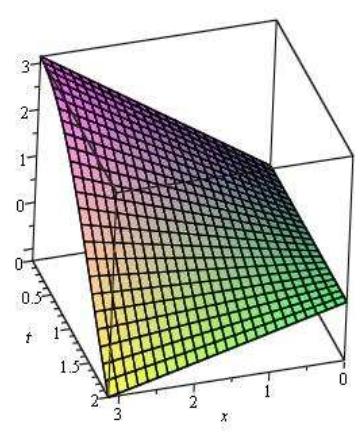

(a)

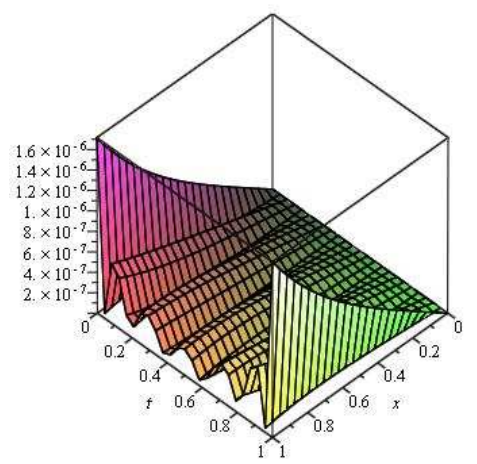

(c)
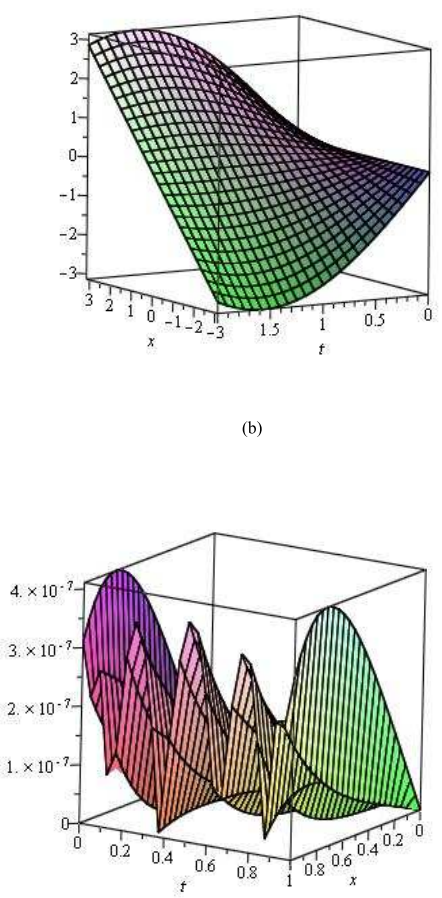

(d)

where $K_{1}$ and $K_{2}$ are known matrices, $A_{1}=P_{y}^{T} C_{1}+V, A_{2}=P_{y}^{T} C_{2}, B_{1}=A_{1}^{T} \tilde{A}_{1}$ and $B_{2}=A_{2}^{T} \tilde{A}_{2}$. Substituting above approximations into system (5.19), leads to the following nonlinear system of the algebraic equations.

$$
\left\{\begin{array}{l}
\Phi^{T}(x, y) C_{1}+\Phi^{T}(x, y) P_{y}^{T} C_{2}-\Phi^{T}(x, y) K_{1}\left(\tilde{B}_{1}-\tilde{B}_{2}\right) \\
P_{x} P_{y} \Phi(x, y)=f_{1}(x, y) \\
\Phi^{T}(x, y) C_{1}+\Phi^{T}(x, y) C_{2}+\Phi^{T}(x, y)\left(P_{y}^{T} C_{1}+V\right)-\Phi^{T}(x, y) P_{y}^{T} C_{2} \\
-\Phi^{T}(x, y) K_{2}\left(\tilde{A}_{1}-\tilde{C}_{2}\right) P_{x} P_{y} \Phi(x, y)=f_{2}(x, y) .
\end{array}\right.
$$

Setting $N=5$ and using the roots of $P_{6}^{(\alpha, \beta)}(x)$ and $P_{6}^{(\alpha, \beta)}(y)$ in the $x$ and $y$-directions, each equation of the system (5.20) is collocated in 36 inner tensor points for different values of parameters $\alpha$ and $\beta$. Hereby, the system (5.20) reduces the problem to solve a system of nonlinear algebraic equations and 72 unknown coefficients are obtained for $\alpha=\beta=0$ by using Newton iterative method. In Figure 2 the exact and approximate solutions are compared for the case $\alpha=\beta=0$ and the absolute and estimate errors functions obtained by the proposed method are also displayed in Figure 2 for $\alpha=\beta=0$. The exact and approximate solutions and their error functions are seen in Figure 3 for various values of $y=0.2,0.4,0.5,0.7,0.9$. 
Figure 3. (a) Comparison of the $u(x, y)$ and $u_{6}(x, y)$, (b) Comparison of the $v(x, y)$ and $v_{6}(x, y),(c)$ Plots of absolute error function $u(x, y),(d)$ Plots of absolute error function $v(x, y)$ for $y=$ $0.2,0.4,0.5,0.7,0.9$ of Example 5.4.
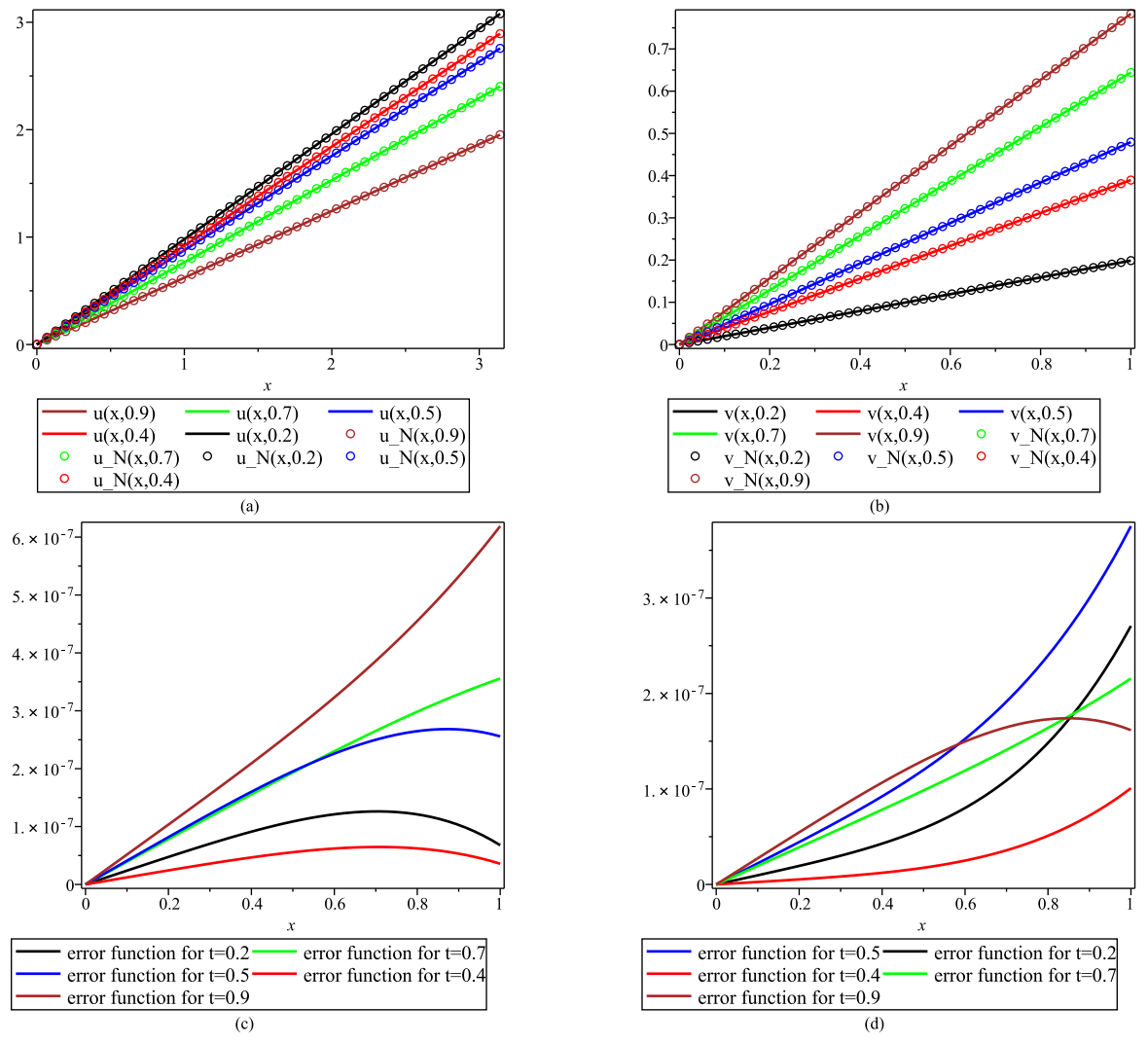

\section{Conclusion}

In this paper, a computational method based on the generalized collocation method was presented for solving some of linear and nonlinear integro-partial differential equations in terms of two variable Jacobi polynomials, by converting them to a linear or nonlinear system of algebraic equations. The illustrative examples with the satisfactory results were achieved to demonstrate the application of this method. The results indicate the proposed approach can be regarded as the simple approach and those are applicable to the numerical solution of these type of equations. It is predicted that the Jacobi collocation method will be a powerful tools for investigating approximate solutions and even analytic to linear and nonlinear functional equations. For numerical purposes the computer programmes have been written in Maple 13.

\section{Acknowledgment}

The authors are deeply grateful to the referee for his careful reading and helpful suggestions on the paper which have improved the paper. 


\section{References}

[1] Bhrawy, A. H. An efficient Jacobi pseudospectral approximation for nonlinear complex generalized Zakharov system, Appl. Math. Comput. 247, 30-46, 2014.

[2] Bhrawy, A. H., Abdelkawy, M. A., Zaky, M. A., and Baleanu, D. Numerical simulation of time variable fractional order mobile-immobile advection-dispersion model, Rom. Rep. Phys. 67 (3), 773-791, 2016.

[3] Bhrawy, A. H., Alofi, A. S., and Ezz-Eldien, S. S. A quadrature tau method for fractional differential equations with variable coefficients, Appl. Math. Lett. 24, 2146-2152, 2011.

[4] Bhrawy, A. H.,Doha, E. H., Baleanu, D., and Ezz-Eldien, S. S. A spectral tau algorithm based on Jacobi operational matrix for numerical solution of time fractional diffusion-wave equations, J. Comput. Phys. 293, Issue C, 142-156, 2015.

[5] Bhrawy, A. H. and Zaky, M. A. A method based on the Jacobi tau approximation for solving multi-term time-space fractional partial differential equations, J. Comput. Phys. 281, 876895, 2015.

[6] Bhrawy, A. H., Zaky, M. A., and Baleanu, D. New Numerical Approximations for SpaceTime Fractional Burgers' Equations via a Legendre Spectral-Collocation Method, Rom. Rep. Phys. 67 (2), 340-349, 2015.

[7] Borhanifar, A. and Abazari, R. Exact solutions for nonlinear Schrödinger equations by differential transformation method, App. Math. Computing. 35 (1), 37-51, 2011.

[8] Borhanifar, A., Jafari, H., and Karimi, S. A. New solitary wave solutions for the bad boussinesq and good boussinesq equations, Numer. Meth. part. Diff. Equ. 25, 1231-1237, 2009.

[9] Borhanifar, A. and Kabir, M. M. New periodic and soliton solutions by application of application of exp-function method for nonlinear evolution equation, Comput. Appl. Math. 229, 158-167, 2009.

[10] Borhanifar, A., Kabir, M. M., and Vahdat, M. New periodic and soliton wave solutions for the generalized zakharov system and $(2+1)$-dimensional nizhnik-novikov-veselov system, Chaos. Solit. Fract. 42, 1646-1654, 2009.

[11] Borhanifar, A. and Sadri, Kh. A new operational approach for numerical solution of generalized functional integro-differential equations, J. Comput. Appl. Math. 279, 80-96, 2015.

[12] Borhanifar, A. and Sadri, Kh. Numerical solution for systems of two dimensional integral equations by using Jacobi operational collocation method, Sohag. J. Math. 1, 15-26, 2014.

[13] Borhanifar, A. and zamiri, A. Application of $\left(\frac{G^{\prime}}{G}\right)$-expansion method for the Zhiber-Shabat equation and other related equations, Math. Comput. Model. 54, 2109-2116, 2011.

[14] Doha, E. H., Abd-Elhameed, W. M., and Youssri, Y. H. Efficient spectral-Petrov-Galerkin methods for the integrated forms of third-and fifth-order elliptic differential equations using general parameters generalized Jacobi polynomials, Appl. Math. Comput. 218, 7727-7740, 2012.

[15] Doha, E. H. and Bhrawy, A. H. An efficient direct solver for multi dimensional elliptic Robin boundary value problems using a Legendre spectral-Galerkin method, Comput. Math. Appl. 64 (4), 558- 571, 2012.

[16] Doha, E. H., Bhrawy, A. H., Abdelkawy, M. A., and Van Gorder, R. A. Jacobi-GaussLobatto collocation method for the numerical solution of $1+1$ nonlinear Schrödinger equations, J. Comput. Phys. 261, 244-255, 2014.

[17] Doha, E. H., Bhrawy, A. H., and Ezz-Eldien, S. S. A new Jacobi operational matrix: An application for solving fractional differential equations, Appl. Math. Model., 36 (10), 49314943, 2012.

[18] Doha, E. H., Bhrawy, A. H., and Ezz-Eldien, S. S. Efficient Chebyshev spectral methods for solving multi-term fractional orders differential equations, Appl. Math. Model. 35, 5662$5672,2011$.

[19] Doha, E. H., Bhrawy, A. H., and Hafez, R. M. A Jacobi-Jacobi dual-Petrov-Galerkin method for third-and fifth-order differential equations, Math. Com. Model. 2011; 53, 1820-1832, 2011.

[20] Doha, E. H., Bhrawy, A. H., and Hafez, R. M. On Shifted Jacobi Spectral Method For HighOrder Multi-Point Boundary Value Problems, Commun. Nonlin. Sci Numer. Simulat. 17 (10), 3802-3810, 2012. 
[21] Guezane-Lakoud, A., Bendjazia, N., and Khaldi, R. Galerkin method applied to telegraph integro-differential equation with a weighted integral condition, Bound. Val. Prob. 102, 1-12, 2013.

[22] Hosseini, S. M. and Shahmorad, S. Numerical solution of a class of integro-differential equations by the Tau method with an error estimation, Appl. Math. Comput. 136, 559-570, 2003.

[23] Karimi Vanani, S. and Aminataei, A. Tau approximate solution of fractional partial differential equations, Comput. Math. Appl. 62, 1075-1083, 2011.

[24] Kreyszig, E. Introduction Functional Analysis with Applications (USA: Wiley, 1978).

[25] Luo, M., Xu, D., Li, L., and Yang, X. Quasi wavelet based on numerical method for Volterra integro-differential equations on unbounded spatial domains, J. Appl. Math. Comput. 227, 509-517, 2014.

[26] Ma, J. Finite element methods for partial Volterra integro-differential equations on twodimensional unbounded spatial domains, Appl. Math. Comput. 186, 598-609, 2007.

[27] Szegö, G. Orthogonal Polynomials (American Mathematical Society, Providence, Rhode Island, 1939).

[28] Tari, A., Rahimi, M. Y., Shahmorad, S., and Talati, F. Development of the Tau Method for the Numerical Solution of Two-dimensional Linear Volterra Integro-differential Equations, Compu. Meth. Appl. Math. 9, 421-435, 2009.

[29] Tari, A. and Shahmorad, S. Differential transform method for the system of two dimensional nonlinear Volterra integro-differential equations, J. Comput. Math. Appl. 61, 2621-2629, 2011. 
